\title{
Optimal portfolios with minimum capital requirements
}

\author{
André A.P. Santos ${ }^{\mathrm{a}, *}$, Francisco J. Nogales ${ }^{\mathrm{b}}$, Esther Ruiz ${ }^{\mathrm{b}}$, Dick Van Dijk ${ }^{\mathrm{c}}$ \\ ${ }^{a}$ Department of Economics, Universidade Federal de Santa Catarina, Campus Universitário Reitor João David Ferreira Lima, 88040-970, Florianópolis, Santa Catarina, Brazil \\ ${ }^{\mathrm{b}}$ Department of Statistics, Universidad Carlos III de Madrid, C/Madrid 126, 28903 Getafe, Madrid, Spain \\ ${ }^{\mathrm{c}}$ Econometric Institute, Erasmus School of Economics, Erasmus University Rotterdam, P.O. Box 17383000 DR, Rotterdam, The Netherlands
}

\section{JEL classification:}

G11

G32

\section{Keywords:}

Convex optimization

Multivariate GARCH

Out-of-sample evaluation

Stress testing

\section{A B S T R A C T}

We propose a novel approach to active risk management based on the recent Basel II regulations to obtain optimal portfolios with minimum capital requirements. In order to avoid regulatory penalties due to an excessive number of Value at Risk (VaR) violations, capital requirements are minimized subject to a given number of violations over the previous trading year. Capital requirements are based on the recent Basel II amendments to account for the 'stressed' VaR, that is, the downside risk of the portfolio under extreme adverse market conditions. An empirical application for two portfolios involving different types of assets and alternative stress scenarios demonstrates that the proposed approach delivers an improved balance between capital requirement levels and the number of VaR exceedances. Furthermore, the risk adjusted performance of the proposed approach is superior to that of minimum VaR and minimum stressed VaR portfolios.

\section{Introduction}

The Basel II framework (Bank for International Settlements, 2006) requires banks to set aside a minimum amount of regulatory capital to cover potential losses arising from their exposure to mar ket risk, credit risk, and operational risk. Market risk is the risk of losses on positions in equities, interest rate related instruments, currencies and commodities due to adverse movements in market prices. The capital requirement (CR) for market risk is based upon estimates of the Value at Risk (VaR), defined as the maximum loss on the bank's positions in these assets that could occur over a given holding period with a specified confidence level. Recent changes in the Basel II regulations establish an additional CR based upon a stressed VaR (sVaR), which reflects the risk on the bank's current portfolio if the relevant market factors were experiencing a period of stress; see Bank for International Settlements (2009).

Basel II allows banks to use 'internal' models to measure their $\mathrm{VaR}$ and sVaR, as an alternative to the standardized approach de scribed in the accord (Hendricks and Hirtle, 1997). This standard ized approach is known to render conservative VaR estimates, leading to excessively high CR. From the banks' perspective this

\footnotetext{
* Corresponding author. Tel.: +55 483721 9458; fax: +55 4837219585.

E-mail addresses: andreportela@cse.ufsc.br (A.A.P. Santos), FcoJavier.Nogales@ uc3m.es (F.J. Nogales), ortega@est-econ.uc3m.es (E. Ruiz), djvandijk@ese.eur.nl (D.V. Dijk).
}

is undesirable given that regulatory capital involves an opportunity cost as it cannot be used for other, profitable purposes. Hence, it is attractive for banks to attempt to lower their capital charges using their own risk management system. The empirical evidence pre sented by Pérignon et al. (2008) suggests that the use of internal models indeed is widespread among large financial institutions.

Although internal risk measurement systems are subject to supervisory approval based on qualitative and quantitative stan dards, banks enjoy a large degree of freedom in devising the pre cise nature of their models. This flexibility does not, however, imply that banks are tempted to pursue the lowest possible VaR estimates. This is due to the fact that the relation between the VaR estimates and capital requirements is non monotonic, as it takes into account not only the magnitude of the VaR but also the number of VaR violations (i.e. actual losses exceeding the $\mathrm{VaR}$ ) in the recent past. Specifically, the regulatory capital required to be held on day $t+1$ is determined as the maximum of the current VaR estimate and the average VaR over the preceding 60 business days multiplied by a scaling factor, that is,

$$
\mathrm{CR}_{t+1} \max \left\{\operatorname{VaR} \$_{t}(h, \alpha),(3+k) \times \overline{\operatorname{VaR} \$_{t, 60}(h, \alpha)}\right\},
$$

where $\operatorname{VaR} \$_{t}(h, \alpha)$ is the estimate at day $t$ of the $\operatorname{VaR}$ for a holding period of $h$ days at confidence level $\alpha \in(0,1)$ and $\overline{\operatorname{VaR} \$_{t, 60}(h, \alpha)}$ $\frac{1}{60} \sum_{j}^{59}{ }_{0} \operatorname{VaR} \$_{t}{ }_{j}(h, \alpha)$. Note that these VaR estimates are expressed in dollar terms, representing the loss that might be incurred on 
the current portfolio; that is, $\operatorname{VaR} \$_{t}(h, \alpha) \quad V_{t}\left(1 \quad e^{\operatorname{VaR}_{t}(h, \alpha)}\right)$ with $V_{t}$ being the current portfolio value and $\operatorname{VaR}_{t}(h, \alpha)$ the VaR in terms of returns. Usually it is the latter VaR that is first obtained from a model for the portfolio return distribution, and we follow this prac tice here. The Basel II accord requires the use of VaR estimates for a holding period $h$ of 10 days at confidence level $\alpha$ of $1 \%$. Moreover, the accord allows the 10 day VaR estimates to be computed from VaR estimates for shorter periods by using the square root of time rule, that is $\operatorname{VaR}_{t}(10, \alpha) \quad \sqrt{ } 10 / h_{V a R}(h, \alpha)$ for some $h<10$, see Bank for International Settlements (2006, paragraph 718 (Lxxvi)). ${ }^{1}$ The penalty or "plus" $k$ in the multiplication factor in (1) ranges between 0 and 1 . Its exact value is determined by the number of $\mathrm{VaR}$ exceedances during the last 250 business days, as shown in Table 1.

During the financial crisis of $2007 / 2008$, losses in most banks' trading books have been substantially larger than the VaR based minimum CR determined according to (1). In response, the Bank of International Settlements (BIS) released a set of modifications to the existing regulatory framework regarding market risk; see Bank for International Settlements (2009). Among the main adjust ments are the introduction of the sVaR and a corresponding new CR formula that leads to higher CR levels. The new CR formula is

$$
\begin{aligned}
\mathrm{CR}_{t+1} & \max \left\{\operatorname{VaR}_{t}(h, \alpha),(3+k) \times \overline{\operatorname{VaR} \$_{t, 60}(h, \alpha)}\right\} \\
& +\max \left\{\operatorname{sVaR} \$_{t}(h, \alpha),(3+k) \times \overline{\operatorname{sVaR} \$_{t, 60}(h, \alpha)}\right\},
\end{aligned}
$$

where $\operatorname{sVaR} \$_{t}(h, \alpha)$ is the estimate at day $t$ of the $\operatorname{sVaR}$ for a holding period of $h$ days at confidence level $\alpha \in(0,1)$ and $\overline{\operatorname{sVaR} \$_{t, 60}(h, \alpha)}$ $\frac{1}{60} \sum_{j{ }_{0}}^{59} \mathrm{SVaR} \$_{t}{ }_{j}(h, \alpha)$. The new regulations state that the backtesting results applicable for calculating the penalty parameter $k$ are based upon estimates of the VaR only and not on the sVaR. Finally, no par ticular methodology is prescribed for computing the sVaR, except that it should reflect the VaR of the bank's current portfolio under extreme adverse market conditions. We discuss in Sections 2.1 and 3.4 alternative approaches to obtain the sVaR.

The expressions for the CR in (1) and (2) seemingly suggest that lower capital charges could be achieved by lower VaR (and sVaR) estimates. This, however, need not be the case as lower VaR esti mates are possibly violated more often, thus increasing the regula tory capital through the effects of the penalty factor $k$. Apart from direct costs due to the larger amount of capital that needs to be put aside, this may also bring indirect costs by damaging the bank's reputation. Both types of costs become particularly severe when the red zone is entered, that is, when ten or more VaR violations occur during a period of 250 business days. In that case, the bank may be forced to adopt the Basel accord's standardized approach for VaR estimation. As noted before, this approach is known to ren der conservative VaR estimates, leading to excessively high CR. In addition, the ban of the bank's internal models obviously has det rimental effects on its reputation.

In practice, banks appear to be wary of being overly optimistic about their level of market risk during tranquil periods. In fact, empirical evidence presented by Berkowitz and O'Brien (2002), Pérignon et al. (2008) and PTrignon and Smith (2010) suggests that they systematically overestimate their VaR. For instance, Berkowitz and O'Brien (2002) document that the number of violations of VaR estimates of six large US banks is usually lower than expected. Similarly, Pérignon et al. (2008) report that for VaR estimates at the $1 \%$ level of the six largest Canadian banks there are only two violations during the 7354 trading days analyzed, whereas the ex pected number is 74. The opposite situation seems to occur in times of stressed market conditions. During the 2007/2008 finan

\footnotetext{
${ }^{1}$ Diebold et al. (1998) and Danielsson and Zigrand (2006) discuss the use of the square root rule.
}

Table 1

Basel II penalty zones.

\begin{tabular}{lll}
\hline Zone & Number of VaR violations & $k$ \\
\hline Green & $0-4$ & 0.00 \\
Yellow & 5 & 0.40 \\
& 6 & 0.50 \\
& 7 & 0.65 \\
& 8 & 0.75 \\
Red & 9 & 0.85 \\
& $>10$ & 1.00 \\
\hline
\end{tabular}

Note: The number of VaR violations is based on the preceding 250 business days.

cial crisis, banks systematically underestimated their VaR and their level of market risk; see Bank for International Settlements (2009). This alternation of over and underestimation of market risk levels may, at least to some extent, be due to the fact that VaR measures typically are calibrated using historical data. Following a period of calm in financial markets, the VaR estimates and the accompany ing CR can decline to low levels, but then they might underesti mate risk during a period of stress that lies ahead. In fact, one of the main motivations for the introduction of the sVaR in the amendments to the Basel II accord is to reduce the procyclicality of the minimum CR. In addition, European regulatory institutions performed a number of stress tests to assess the resilience of the banking system to absorb shocks on credit, market and sovereign risks and introduced further modifications in the regulatory frame work; see Committee of European Banking Supervisors (2010).

The exaggeration of banks' own level of risk during normal times implies an excessive amount of regulatory capital, directly affecting the profitability of the bank. Another, at least as undesir able consequence is that such banks appear more risky than they actually are, thus generating reputational concerns about their risk management systems. Similarly, the underestimation of banks' own level of risk during times of stressed market conditions may lead to insufficient amount of regulatory capital to cover potential losses in the trading book, thus increasing the risk of bankruptcy. This affects investors' perception and can induce underinvestment in VaR overstating and VaR understating banks. Indeed, Jorion (2002) shows that VaR disclosures are informative about the future variability in trading revenues, thus corroborating the idea that analysts/investors may be using the VaR measures to support investment decisions.

In this paper we put forward a novel portfolio construction methodology to overcome the drawbacks of both over and under statement of a bank's VaR. Specifically, we propose to determine optimal portfolio weights by directly minimizing the CR subject to a restriction on the number of VaR violations during the preced ing year ( 250 business days). Implicitly, our approach aims to find the optimal balance between the level of VaR measures and the number of VaR violations, thus leading to the lowest possible level of CR.

Although minimizing $\mathrm{CR}$ is an important criterion to take into account, in real world situations portfolio managers and investors traditionally decide upon their asset allocations by considering standard performance measures, such as expected returns or Sharpe ratios. In addition, portfolio weights often are restricted in order to avoid shortselling or to limit the exposure to individual assets. For this reason, we consider a general formulation of the portfolio construction problem in which the optimal portfolio com position is found by minimizing the level of CR subject also to a gi ven (i.e. user specified) target performance and to direct constraints on the portfolio weights.

We apply the proposed methodology to two different asset portfolios: (i) a mixed portfolio composed of 30 futures on a variety of assets including equities, bonds, commodities and currencies, and (ii) an equity portfolio comprising 48 US industry indices. 
The minimum capital requirement (MCR) portfolio is compared to various benchmark portfolios, including the minimum VaR portfo lio (Alexander and Baptista, 2002), the minimum sVaR portfolio, and the equally weighted $(1 / N)$ portfolio. In our empirical analysis we pay particular attention to the consequences of the introduc tion of the sVaR based CR. For this purpose, in addition to 'normal' market conditions we consider several alternative, realistic scenar ios in which expected returns, volatilities and cross correlations are modified to reflect a stressed environment. Furthermore, we consider different models for obtaining forecasts of expected returns, volatilities and correlations, which are crucial inputs for the asset allocation decisions. We also examine the robustness of our results to the specific restrictions imposed on the portfolio's target rate of return and the re balancing frequency.

The results for the futures portfolio indicate that our approach delivers lower CR levels in comparison to the benchmark portfo lios. For the portfolios of sector indices, the novel portfolio construction approach delivers a better balance between CR levels and the number of VaR violations, as it yields a lower average number of VaR exceedances. For both data sets, we find that the number of VaR violations under the MCR portfolio policy does not enter the red zone in any of the (normal and) stress scenarios regardless of the specifications used for forecasting volatilities and correlations. This is in sharp contrast to the benchmark portfolios, for which we frequently find more than ten VaR violations. Finally, the performance of the MCR portfolios in terms of risk adjusted returns and portfolio turnover is generally superior to the mini mum VaR and minimum sVaR portfolios.

Our proposed methodology differs in important ways from pre vious, related research. First, in order to achieve the goal of lower $\mathrm{CR}$, one possibility is to develop a VaR model that delivers lower levels of capital charges, as proposed recently by McAleer et al. (2010), for instance. Using the terminology of Christoffersen (2009), this approach can be considered a risk measurement or passive risk management approach, since it is applied to a given (i.e. predetermined) portfolio composition. Alternatively, in this paper, we propose to perform active risk management by deciding on the portfolio allocations themselves to attain lower levels of CR.

Second, portfolios with low levels of CR may be obtained by imposing constraints on the amount of $\mathrm{CR}$ or on the portfolio VaR, as in Sentana (2003), Cuoco and Liu (2006) and Alexander et al. (2007). In our approach, the level of CR plays a much more central role as it is taken to be the objective function that should be minimized.

The remainder of the paper is organized as follows. In Section 2 we describe the procedure to obtain optimal portfolios with mini mum $C R$ subject to restrictions on the number of VaR violations. In Section 3 we present the empirical applications. We conclude in Section 4

\section{Portfolios with minimum capital requirements}

The main ingredient required to obtain optimal portfolios with minimum capital requirements is a measure of the VaR and of the sVaR. Therefore, in Section 2.1, we first describe the procedure for obtaining VaR and sVaR estimates considered in this paper. In Sec tion 2.2, we then develop the optimization problem that leads to the construction of MCR portfolios.

\subsection{VaR and sVaR estimation}

Denote by $R_{t+h}=\left(r_{1, t+h}, \ldots, r_{N, t+h}\right)^{\prime}$ the vector of $h$ period returns (between $t$ and $t+h$ ) of the $N$ assets included in the portfolio. The portfolio return is given by $r_{p, t+h} \quad w_{t}^{\prime} R_{t+h}$, where $w_{t}$ is the vec tor of portfolio weights to be determined at time $t$. The portfolio
VaR at time $t$ for a given holding period $h$ and confidence level $\alpha$ is given by the $\alpha$ quantile of the conditional distribution of the portfolio return. Thus, $\operatorname{VaR}_{t}(h, \alpha) \quad F_{p, t+h}^{1}(\alpha / 100)$, where $F_{p, t+h}^{1}$ is the inverse of the cumulative distribution function of $r_{p, t+h}$. Throughout the paper we focus on the portfolio VaR for a holding period of $h=1$ day at $\alpha=1 \%$. The latter is the relevant confidence level that banks must adopt in computing their risk exposure. As mentioned before, the Basel accord requires the use of VaR esti mates for a holding period $h$ of 10 days, but it allows these to be computed from VaR estimates for shorter periods by using the square root of time rule. Therefore, from now on, we omit the arguments $h$ and $\alpha$ from the definition of the VaR.

When the distribution of returns is expressed in terms of its two first conditional moments, the portfolio return can be represented as

$r_{p, t+1} \quad \mu_{p, t+1}+\sigma_{p, t+1} z_{p, t+1}$,

where $\mu_{p, t+1}$ and $\sigma_{p, t+1}$ are the conditional mean and standard devi ation of the portfolio return, given by

$\mu_{p, t+1} \quad w_{t}^{\prime} \mu_{t+1}$

and

$\sigma_{p, t+1}^{2} \quad w_{t}^{\prime} H_{t+1} w_{t}$

where $\mu_{t+1}=\mathrm{E}\left[R_{t+1} \mid R_{1}, \ldots, R_{t}\right]$ is the $N \times 1$ vector of conditional mean returns for the $N$ individual assets and $H_{t+1}$ is their $N \times N$ condi tional covariance matrix, $H_{t+1}=\mathrm{E}\left[\left(\begin{array}{lll}R_{t+1} & \left.\mu_{t+1}\right)^{\prime}\left(R_{t+1}\right. & \mu_{t+1}\end{array}\right) \mid R_{1}, \ldots\right.$ ,$\left.R_{t}\right]$. The standardized unexpected returns $z_{p, t+1}$ in (3) are indepen dent and identically distributed with mean equal to zero and unit variance, i.e. $\mathrm{E}\left[z_{p, t+1} \mid R_{1}, \ldots, R_{t}\right]=0$ and $\mathrm{E}\left[z_{p, t+1}^{2} \mid R_{1}, \ldots, R_{t}\right] \quad 1$ for all $t$. The portfolio VaR is then given by

$\mathrm{VaR}_{t+1} \quad \mu_{p, t+1}+\sigma_{p, t+1} q$

where $q$ is the $\alpha$ quantile of the distribution of $z_{p, t+1}$.

The novel CR specification in (2) requires the estimation of the sVaR. As mentioned before, the sVaR is similar to the 'normal' VaR, except that it should measure the risk of extreme losses if the rel evant market factors were experiencing a period of stress. Accord ingly we define the sVaR as:

$\operatorname{sVaR}_{t} \tilde{\mu}_{p, t+1}+\tilde{\sigma}_{p, t+1} q$,

where $\mu_{p, t+1} \quad w^{\prime} \mu_{t+1}, \sigma_{p, t+1} \quad\left(w^{\prime} \widetilde{H}_{t+1} w\right)^{1 / 2}$, and $\mu$ and $\widetilde{H}$ are the vector of stressed conditional expected returns and the stressed conditional covariance matrix, respectively. The amendments to the Basel accord do not provide specific implementation details concerning the stress scenarios, except that they should typically involve lower expected returns, higher volatilities and more ex treme correlations. In Section 3.4 we discuss the specification of alternative stress scenarios used in the empirical analysis.

The VaR definition in (6) requires estimates of the conditional expected returns $\mu_{t+1}$, the conditional covariance matrix $H_{t+1}$ and the quantile $q$ or, more generally, the distribution of the standard ized unexpected returns. For each of these three inputs, both non parametric and parametric specifications may be adopted. Given that in this paper we focus on high dimensional portfolios consist ing of a large number of assets $N$, parametric specifications may be more appropriate. In this case, the expected returns may be ob tained from linear (vector) autoregressive [(V)AR] models as well as nonlinear models (Carriero et al., 2009; Pesaran et al., 2009; DeMiguel et al., 2010). Similarly, alternative specifications for the conditional covariance matrix $H_{t+1}$ can be considered, including multivariate GARCH models (see Bauwens et al. (2006) and Silvennoinen and TerSsvirta (2009) for comprehensive reviews), stochastic volatility models (Harvey et al., 1994; Aguilar and West, 2000; Chib et al., 2009), as well as realized covariance matrices 
based on high frequency intraday data (De Pooter et al., 2008; Barndorff Nielsen et al., 2008). Finally, the models for the conditional mean and variance are usually estimated by maximum likelihood (or comparable methods), which requires assuming a particular distribution for $z_{p, t+1}$, such as the normal or the Student's $t$ distribution. This distribution may also be considered in order to obtain the quantile $q$ in (6). For instance, when assuming normality of $z_{p, t+1}, q=2.33$ for $\alpha=1 \%$. See Santos et al. (2009) for an empirical comparison among alternative procedures for computing the inverse of the cumulative distribution function of the portfolio returns.

Finally, it is worth noting that the MCR portfolio construction methodology developed in the remainder of this Section is inde pendent of the method used to obtain the VaR and sVaR measures. However, and perhaps obviously, we may expect that more accu rate modeling of the expected returns and conditional covariance matrix leads to improved portfolio characteristics.

\subsection{MCR portfolios}

The problem of constructing an MCR portfolio consists of find ing the vector of portfolio weights $w$ that minimizes the capital charges subject to a restriction on the number of VaR exceptions during the previous 250 trading days and other constraints. We now describe the objective function as well as the restrictions in volved in the optimization problem in more detail.

\subsubsection{Objective function}

The objective function for constructing an MCR portfolio con sists of minimizing the amount of regulatory capital given by the 'original' Basel II formulation in (1) or by the 'new' specification in (2). To save space we focus on the latter, noting that it is straightforward to formulate an analogous objective function using the $\mathrm{CR}$ formula in (1). For the sake of convenience, we rephrase the function given in (2) in terms of the VaR and the sVaR expressed in portfolio returns. Using the VaR and sVaR definitions in (6) and (7) and the expressions for the conditional mean and variance of the portfolio returns in (4) and (5), we can write the objective function as

$$
\begin{aligned}
& \underset{w}{\operatorname{minimize} \max }\left\{\left(w^{\prime} \mu_{t+1}+\left(w^{\prime} H_{t+1} w\right)^{1 / 2} q\right), \frac{(3+k)}{60} \sum_{j 0}^{59}\left(w^{\prime} \mu_{t+1} j\right.\right. \\
& \left.\left.+\left(w^{\prime} H_{t+1} j w\right)^{1 / 2} q\right)\right\}+\max \left\{\left(w^{\prime} \tilde{\mu}_{t+1}+\left(w^{\prime} \widetilde{H}_{t+1} w\right)^{1 / 2} q\right),\right. \\
& \left.\quad \frac{(3+k)}{60} \sum_{j 0}^{59}\left(w^{\prime} \tilde{\mu}_{t+1} j+\left(w^{\prime} \widetilde{H}_{t+1} j w\right)^{1 / 2} q\right)\right\} .
\end{aligned}
$$

In the first (second) term of (8), we take the maximum of minus the current VaR (sVaR) estimate and the average over the previous 60 business days. Note that for computing the average VaR and sVaR over the previous 60 business days we need the historic one step ahead forecasts for (i) the conditional mean and for the stressed conditional mean, $\mu_{t+1} j_{j}$ and $\mu_{t+1} j$, and (ii) the conditional covariances and the stressed conditional covariances, $H_{t+1} j$ and $\widetilde{H}_{t+1 j}$, for $j=0, \ldots, 59$. Even more important to note is that the average VaR and sVaR are hypothetical, in the sense that they are based on the portfolio with the weights that are currently deter mined for day $t+1$.

\subsubsection{Restriction on the number of VaR violations}

A VaR violation occurs when the portfolio return on a given trad ing day falls below the VaR estimate. This can be characterized by means of an indicator function, $\mathbf{1}\left(w^{\prime} R_{t+1}<w^{\prime} \mu_{t+1}+\left(w^{\prime} H_{t+1} w\right)^{1 / 2} q\right)$, which takes the value 1 when the argument is true, i.e. when a $\mathrm{VaR}$ violation occurs on day $t+1$. We restrict the number of VaR exceedances over the last 250 trading days to be less than or equal to a certain threshold $\delta$, that is, we impose the restriction

$$
\sum_{j 1}^{250} \mathbf{1}\left(w^{\prime} R_{t+1}{ }_{j}<w^{\prime} \mu_{t+1}{ }_{j}+\left(w^{\prime} H_{t+1}{ }_{j} w\right)^{1 / 2} q\right) \leqslant \delta .
$$

The value of $\delta$ can be chosen by taking into consideration the penalties reported in Table 1. For instance, if we intend to avoid the number of VaR violations reaching the red zone, we should set $\delta=9$. Similar to the average VaR and sVaR over the previous 60 business days in (8), the number of VaR violations over the previous 250 days in (9) is hypothetical, in the sense that it is based on the portfolio with the weights that are currently deter mined for day $t+1$. $^{2}$ It is also important to note that we include a restriction on the number of VaR violations and not on the num ber of sVaR violations. This is in line with the current regulations, which establish that backtesting results applicable for calculating the penalty parameter $k$ in (2) are based upon estimates of the VaR only and not on the sVaR; see Bank for International Settle ments (2009).

\subsubsection{Target performance}

In many practical situations involving portfolio selection, inves tors and portfolio managers are interested in achieving a certain target performance. For that purpose, we incorporate the following restriction on the expected portfolio returns:

$w^{\prime} \mu_{t+1} \geqslant \Xi$,

where $\Xi$ denotes the desired target performance. Note that alterna tive specifications for restrictions on the target performance can be considered, such as a constraint on the Sharpe ratio, on the portfolio turnover, or on the tracking error; see Cornuejols and Tütüncü (2007).

\subsubsection{Constraints on the portfolio weights}

Finally, restrictions often are imposed on the portfolio weights to avoid short selling or to achieve a minimum diversification le vel. Previous research has shown that imposing such constraints may substantially improve performance, mostly by reducing risk, see Jagannathan and Ma (2003), among others. For this reason, we allow for a general set of constraints on the portfolio weights, formulated as

$w \in \Omega$,

where $\Omega$ represents the set of allowable portfolio weights as de fined by the specific restrictions that are imposed. For instance, if short selling is to be avoided, we may specify the restriction $w \geqslant 0$. Similarly, a certain level of diversification may be guaran teed by imposing an upper bound on the individual portfolio weights, i.e. $w_{i} \leqslant u$ for some $0<u<1$. $^{3}$ Finally, we may achieve full investment by restricting the portfolio weights to sum up to 1, i.e. $l^{\prime} w=1$, where $l$ is a vector of ones.

The optimization problem as formulated in (8) (11) is very gen eral as it allows (i) different econometric specifications for the ex pected returns and for the conditional covariance matrix, (ii) different threshold levels for the maximum number of VaR viola tions, (iii) different types of restrictions on the target performance, (iv) alternative stress scenarios for the computation of the sVaR, and (v) various different constraints on the portfolio weights.

\footnotetext{
2 Of course, the ex-post evaluation of the portfolio in terms of the number of VaR violations and capital requirements is based on actual portfolios, therefore in accordance to the criteria established by Basel II. Further implementation details are discussed in Section 3.5.

${ }^{3}$ DeMiguel et al. (2009a) recently proposed a unifying approach based on constraints of the portfolio norms that nests several commonly applied restrictions as special cases, including the no-shortselling and diversification constraints.
} 
Albeit very general, the formulation in (8) (11) has a major short coming: the objective functions in (8), and the restriction on the number of VaR violations in (9) are both discontinuous and non convex due to the presence of the max operator and the indicator function, respectively. Note that the non convexity imposes impor tant difficulties in terms of computational effort and a potential problem of local minima; see Nocedal and Wright (1999), Boyd and Vandenbergue (2004). For this reason we next formulate a convex and continuous approximation to the original problem for which a highly accurate solution can be obtained with low compu tational effort.

\subsection{A convex and continuous reformulation}

\subsubsection{Reformulating the objective function}

In order to obtain a continuous and smooth objective function, (8) can be reformulated by introducing artificial variables to get rid of the max operator. Specifically, the objective function in (8) can be equivalently expressed as the following linear optimization problem:

$$
\begin{aligned}
\underset{w, v_{1}, v_{2}}{\operatorname{minimize}} & v_{1}+v_{2} \\
\text { subject to }: & v_{1} \geqslant\left(w^{\prime} \mu_{t+1}+\left(w^{\prime} H_{t+1} w\right)^{1 / 2} q\right) \\
& v_{1} \geqslant \frac{(3+k)}{60} \sum_{j 0}^{59}\left(w^{\prime} \mu_{t+1}{ }+\left(w^{\prime} H_{t+1} j w\right)^{1 / 2} q\right) \\
& v_{2} \geqslant\left(w^{\prime} \tilde{\mu}_{t+1}+\left(w^{\prime} \widetilde{H}_{t+1} w\right)^{1 / 2} q\right) \\
& v_{2} \geqslant \frac{(3+k)}{60} \sum_{j 0}^{59}\left(w^{\prime} \tilde{\mu}_{t+1} j+\left(w^{\prime} \widetilde{H}_{t+1} j w\right)^{1 / 2} q\right),
\end{aligned}
$$

thus yielding a continuous and convex expression. Note that the attractiveness of the reformulation in (12) is that it replaces the minimization of a nonlinear, non smooth objective function by the minimization of a smooth, linear objective function with convex constraints (Nocedal and Wright, 1999).

\subsubsection{Reformulating the restriction on the number of VaR violations}

Due to the presence of an indicator function, the restriction on the number of VaR violations in (9) is non differentiable, discontin uous and non convex. We propose a convex approximation by elim inating the indicator function while keeping its argument, which leads to

$$
\frac{1}{250} \sum_{j 1}^{250}\left(w^{\prime} \mu_{t+1} j_{j}+\left(w^{\prime} H_{t+1}{ }_{j} w\right)^{1 / 2} q \quad w^{\prime} R_{t+1} j\right)<\tilde{\delta}
$$

where $\delta$ is a parameter that must be calibrated in order to achieve the desired result regarding the number of VaR violations over the last 250 observations. The procedure to calibrate this parameter is detailed in Section 3.5. Note that under this approx imation, our displeasure regarding a VaR violation grows as the constraint becomes "more violated". In other words, this approx imation implies that VaR violations of greater magnitude are more penalized than those of less magnitude, which makes sense from a practical point of view. Note that this feature is not cap tured by the indicator function as, in that case, VaR exceedances of smaller magnitude have the same importance as those of greater magnitude. Moreover, the proposed approximation is the best convex approximation to (9); see Boyd and Vandenber gue (2004).

In sum, based on the reformulation of the objective function in (12) and of the constraint on the number of VaR violations in (13), the optimization problem based on (8) (11) admits the following convex reformulation:

$$
\begin{array}{ll}
\underset{w, v_{1}, v_{2}}{\operatorname{minimize}} & v_{1}+v_{2} \\
\text { subject to }: & v_{1} \geqslant\left(w^{\prime} \mu_{t+1}+\left(w^{\prime} H_{t+1} w\right)^{1 / 2} q\right) \\
& v_{1} \geqslant \frac{(3+k)}{60} \sum_{j 0}^{59}\left(w^{\prime} \mu_{t+1}+\left(w^{\prime} H_{t+1} j w\right)^{1 / 2} q\right) \\
& v_{2} \geqslant\left(w^{\prime} \tilde{\mu}_{t+1}+\left(w^{\prime} \widetilde{H}_{t+1} w\right)^{1 / 2} q\right) \\
& v_{2} \geqslant \frac{(3+k)}{60} \sum_{j 0}^{59}\left(w^{\prime} \tilde{\mu}_{t+1}+\left(w^{\prime} \widetilde{H}_{t+1} j\right)^{1 / 2} q\right) \\
& \frac{1}{250} \sum_{j 1}^{250}\left(w^{\prime} \mu_{t+1}+\left(w^{\prime} H_{t+1} j w\right)^{1 / 2} q \quad w^{\prime} R_{t+1} j\right)<\tilde{\delta} \\
& w^{\prime} \mu_{t+1} \geqslant \Xi \\
& w \in \Omega .
\end{array}
$$

Note that the optimization problem (14) is a second order cone formulation (Nocedal and Wright, 1999; Boyd and Vandenbergue, 2004; Grant and Boyd, 2008). Therefore, the problem can be accu rately solved in practice with low computational effort.

A final, technical comment about the optimization problem in (14) concerns the penalty factor $k$. Since the MCR portfolios are dy namic in the sense that the optimal portfolio weights have to be re calculated on a daily basis, the penalty $k$ also should be updated based on the actual number of VaR violations over the previous 250 trading days. This means that a minimum of 250 realizations of the MCR portfolio returns and corresponding VaRs are required to start evaluating and updating $k$. In order to be conservative, dur ing the first 250 observations we set $k=1$ in the computation of the capital requirements, which is the highest value that the pen alty factor can take. After the 250th trading day, we update $k$ according to the values presented in Table 1. Moreover, to ensure a consistent portfolio evaluation we focus on the capital require ments and the number of VaR violations obtained after the 250th day.

\section{Empirical application}

We evaluate the performance of the MCR strategy for two port folios with different types of assets. In the optimization problem in (14), we adopt a daily target portfolio return of $\Xi=4 \mathrm{bp}$, corre sponding to an annual target return of $10 \%$. Furthermore, we focus on the case in which only long positions are allowed by imposing a no shortselling restriction, i.e. $w \geqslant 0$.

\subsection{Data sets}

We consider portfolios constructed from two sets of different types of assets. ${ }^{4}$ The first set consists of 30 futures contracts on equity indices (S\&P500, NASDAQ, DJIA, Canada 60, FTSE, CAC, DAX, IBEX, MIB, Nikkei, Hang Seng, SGX, Bovespa, IPC), 10 year gov ernment bonds (US, UK, Germany, and Japan), currencies (Euro, British Pound, Japanese Yen, Canadian Dollar, Swiss Franc, Austra lian Dollar, Mexican Peso and Brazilian Real) and commodities (gold, silver, wheat, and crude). For each contract, we measure re turns in Dollars, and implement appropriate adjustments for roll overs from one futures contract to the next. The second set of assets comprises 48 industry portfolios of US stocks. ${ }^{5}$ For the two sets of assets we obtain daily observations from March 1, 2000 until July

\footnotetext{
${ }^{4}$ We have considered a third data set consisting of all stocks that belonged to the S\&P100 index during the complete sample period. This yields a total of 81 stocks. The results are very similar to those obtained with the US industry portfolios and therefore are not reported to save space.

5 This data set was obtained from the web page of Kenneth French (http:// mba.tuck.dartmouth.edu/pages/faculty/ken.french/).
} 
31,2008 . Returns are computed as the differences in log prices. The effective sample sizes are equal to 2194 and 2156 observations, respectively.

\subsection{Expected returns and conditional covariances}

Computing a VaR measure for a given portfolio requires estimates of the expected returns and the conditional covariance matrix of the included assets; see (6). In our empirical application these inputs are obtained from multivariate parametric models.

Expected returns are obtained from a $\operatorname{VAR}(1)$ model for the return vector $R_{t+1}$,

$R_{t+1} \quad C+\Phi R_{t}+\varepsilon_{t+1}$,

where $C$ is an $N \times 1$ vector of constants, $\Phi$ is the $N \times N$ autoregres sive matrix, and $\varepsilon_{t+1}$ is a vector of shocks (or unexpected returns), which are assumed to be temporally uncorrelated and conditionally Normal distributed with a positive definite conditional covariance matrix $H_{t+1}$.

We consider two of the most popular specifications for the con ditional covariance matrix: the Risk Metrics model and the dy namic conditional correlation (DCC) model of Engle (2002). Moreover, we also consider the (unconditional) shrinkage estima tor of the sample covariance matrix proposed by Ledoit and Wolf (2003), motivated by its ability of dealing with the estimation error in large covariance matrices.

Widely used by practitioners, the Risk Metrics (RM) approach consists of an exponentially weighted moving average scheme to model conditional covariances. In this approach, the conditional covariance matrix is given by

$H_{t+1} \quad\left(\begin{array}{ll}1 & \lambda\end{array}\right) R_{t} R_{t}^{\prime}+\lambda H_{t}$,

with the recommended value for the model parameter for daily returns being $\lambda=0.94$.

Time varying conditional correlation models are currently one of the most promising alternatives to model and forecast condi tional covariances; see Engle and Sheppard (2001) for comprehen sive theoretical and empirical analysis. One of their greatest advantages is that they have a smaller number of parameters than traditional multivariate models, such as VEC and BEKK models and, therefore, can be applied to problems involving a large number of assets. DCC models are based on the decomposition of the condi tional covariance matrix $H_{t+1}$ into conditional standard deviations and correlations, that is

$H_{t+1} \quad D_{t+1} P_{t+1} D_{t+1}$,

where $D_{t+1} \operatorname{diag}\left(\sqrt{ } h_{1, t+1}, \ldots, \sqrt{ } h_{N, t+1}\right)$ with diag ( ) being the operator that transforms a $N \times 1$ vector into a $N \times N$ diagonal ma trix. The conditional variances $h_{j, t+1}, j=1, \ldots, N$, are assumed to fol low a standard univariate $\operatorname{GARCH}(1,1)$ model. $P_{t+1}$ is a symmetric positive definite conditional correlation matrix with elements $\rho_{i j, t+1}$, where $\rho_{i i, t+1}=1, i, j=1, \ldots, N$. In the DCC model the conditional cor relation $\rho_{i j, t+1}$ is given by

$\rho_{i j, t+1} \frac{q_{i j, t+1}}{\sqrt{ } q_{i i, t+1} q_{j j, t+1}}$,

where $q_{i j, t+1}, i, j=1, \ldots, N$, are collected into the $N \times N$ matrix $Q_{t+1}$, which is assumed to follow GARCH type dynamics,

$Q_{t+1} \quad\left(\begin{array}{lll}1 & \alpha & \beta\end{array}\right) \bar{Q}+\alpha z_{t} z_{t}^{\prime}+\beta Q_{t}$,

where $z_{t}=\left(z_{1 t}, \ldots, z_{N t}\right)$ with elements $z_{i t} \quad \varepsilon_{i t} / \sqrt{ } h_{i t}$ being the standardized unexpected returns, $\bar{Q}$ is the $N \times N$ unconditional covariance matrix of $z_{t}$ and $\alpha$ and $\beta$ are non negative scalar param eters satisfying $\alpha+\beta<1$. $^{6}$

Our third and final approach to model the covariance matrix is the shrinkage estimator of Ledoit and Wolf (2003) (LW). Shrinkage estimators are becoming very popular in the portfolio construction literature due to their ability to reduce the estimation error in large covariance matrices. For instance, Ledoit and Wolf (2003, 2004) report improved results in terms of portfolio performance when the shrinkage estimator is used vis à vis traditional estimators such as the sample covariance matrix. In this paper, we consider the shrinkage estimator proposed by Ledoit and Wolf (2003), which is defined as an optimally weighted average of the sample covariance matrix and the covariance matrix based on Sharpe (1963) single index model. The intuition behind this shrinkage estimator is to come up with an optimal convex combination between an unbiased covariance matrix estimator that may be subject to substantial estimation error (i.e. the sample covariance matrix) and another estimator that possibly is biased but has considerably less estima tion error (i.e. the covariance matrix from the single factor model). In this model the returns of asset $i$ are described by:

$r_{i t} \quad a_{i}+b_{i} r_{m t}+v_{i, t}$,

where $r_{m t}$ is the market portfolio return. ${ }^{7}$ The residuals $v_{i}$ are as sumed to be uncorrelated with market returns and to exhibit no se rial correlation. The covariance matrix $F$ of the returns $R_{t}$ implied by this model is:

F $\quad \sigma_{m}^{2} b b^{\prime}+\Delta$,

where $\sigma_{m}^{2}$ is the variance of the market returns, $b$ is the vector of slopes or factor loadings, and $\Delta$ is a diagonal matrix containing vari ances of the residuals $v_{t}$. The shrinkage estimator of Ledoit and Wolf (denoted by $H_{L W}$ ) is then defined as

$H_{L W} \quad \psi F+(1 \quad \psi) S$,

where $\psi$ is the shrinkage intensity and $S$ is the sample covariance matrix. A closed form solution for the optimal shrinkage intensity (minimizing the distance between the true and estimated covari ance matrices based on the Frobenius norm) is provided by Ledoit and Wolf (2003). ${ }^{8}$

\subsection{Benchmark portfolios}

We consider three alternative benchmarks for the purpose of comparison with the proposed MCR portfolios. The first benchmark

\footnotetext{
${ }^{6}$ The parameters of the DCC model are usually estimated using the two-step procedure proposed by Engle and Sheppard (2001) and Sheppard (2003). However, Engle et al. (2008) point out that when the dimension of the portfolio increases, this two-step estimator can be severely downward biased due to an undiagnosed incidental parameter problem. Therefore, in this paper, we estimate the parameters of the DCC model by the composite likelihood (CL) estimator proposed by Engle et al. (2008). Essentially, this method is based on the decomposition of the original estimation problem into many small subproblems in such a way that the composite likelihood is constructed by summing up the quasi-likelihood of subset of assets. By summing up over many subsets, the CL estimator does not require the inversion of large covariance matrices. In the case where all distinct pairs of the returns in the system are used, the CL involves $O\left(K^{2}\right)$ calculations, which is much smaller than the $O\left(K^{3}\right)$ implied in the maximization of the standard likelihood function, where $K$ is the number of unique pairs of data. Consequently, the CL estimator is much faster. Furthermore, Engle et al. (2008) show that the CL estimator is not affected by the incidental parameter problem. Instead of all distinct pairs, in this work, we implement the CL estimator using all contiguous pairs of data, which implies $O(K)$ calculations; the extensive Monte Carlo results reported in Engle et al. (2008) show that the choice of contiguous pairs can be successfully applied in problems involving hundreds of assets.

7 We follow Ledoit and Wolf (2003) and use an equally weighted combination of the assets belonging to the portfolio under consideration as the 'market portfolio'.

${ }^{8}$ Code for computing the optimal shrinkage intensity is available at http://
} www.iew.uzh.ch/institute/people/wolf/publications.html. 
is a minimum VaR (Min VaR) portfolio. This is obtained from an optimization problem in which the investor wishes to perform ac tive portfolio management by minimizing the portfolio VaR subject to a target return and possible other restrictions. ${ }^{9}$ The Min VaR optimization problem is given by

$$
\begin{gathered}
\underset{w}{\operatorname{minimize}} \quad\left(w^{\prime} \mu_{t+1}+\left(w^{\prime} H_{t+1} w\right)^{1 / 2} q\right) \\
\text { subject to : } \quad w^{\prime} \mu_{t+1} \geqslant \Xi \\
\\
w \in \Omega .
\end{gathered}
$$

In (23), we adopt the same target return and the no shortselling restriction used to obtain MCR portfolios.

The second benchmark portfolio is a minimum sVaR (Min sVaR) portfolio. This is obtained from an optimization problem analogous to (23), but replacing the conditional mean and the con ditional covariance in the objective function by their stressed counterparts $\mu_{t+1}$ and $\widetilde{H}_{t+1}$. In the resulting Min sVaR optimization problem we adopt the same target return and the no shortselling constraint used to obtain MCR portfolios.

As a third benchmark, we consider the equally weighted (or $1 / N$ ) portfolio, which has been extensively studied in the empirical liter ature. For instance, DeMiguel et al. (2009b) find that the $1 / N$ port folio outperforms (in terms or Sharpe ratio and turnover) 14 widely used portfolio strategies, such as mean variance and mini mum variance. Therefore, it seems natural to compare our proposed portfolio against this simple but powerful portfolio in which all the assets have the same weight. We note that the $1 / N$ portfolio returns are independent of the method used to model and forecast the ex pected returns and the conditional covariance matrix. On the other hand, the VaR estimate, the level of capital requirements, and the number of VaR violations are affected by these methods.

\subsection{Stress scenarios}

The sVaR measures the risk of extreme losses if the relevant market factors were experiencing a period of stress. As mentioned before, the amendments to the Basel accord do not provide specific implementation details concerning the stress scenarios, except that they should typically involve lower expected returns, higher volatilities and more extreme correlations in agreement with empirical evidence; see Alexander (2009, Chapter IV.7). We con sider three alternative stress scenarios that reflect the impact of relevant changes or 'haircuts' to the conditional moments of the asset returns on the estimation of the sVaR. ${ }^{10}$

\subsubsection{Stress scenario 1 (expected returns)}

In this scenario, we apply a haircut to the conditional expected return, i.e. $\mu_{t} \quad \mu_{t} \quad \Delta_{\mu}$, where $\Delta_{\mu}>0$ is the haircut level for the expected return. In this case, we have $\mathrm{sVaR}_{t} w^{\prime} \mu_{t}+$ $\left(w^{\prime} D_{t} P_{t} D_{t} w\right)^{1 / 2} q$.

\subsubsection{Stress scenario 2 (expected returns and volatilities)}

In this case, we lower the conditional expected returns as in scenario 1 but also apply a haircut to the volatilities, i.e. $\mu_{t} \quad \mu_{t} \quad \Delta_{\mu}$ and $\widetilde{D}_{t} \quad D_{t}+\Delta_{D}$, where $\Delta_{D}>0$ is the haircut level for the volatilities. In this case, we have $\operatorname{sVaR}_{t} w^{\prime} \mu_{t}+$ $\left(w^{\prime} \widetilde{D}_{t} P_{t} \widetilde{D}_{t} w\right)^{1 / 2} q$.

\footnotetext{
9 The properties of the Min-VaR portfolio have been extensively studied by Alexander and Baptista (2002). It is shown that the solution to the VaR minimization problem is always distinct from the solution to the variance minimization problem. Moreover, the Min-VaR portfolio at the $99 \%$ confidence level is a mean-variance efficient portfolio with expected returns greater than the expected return of the minvariance portfolio.

${ }^{10}$ In unreported results, we consider a fourth stress scenario in which only correlations are stressed. The results are similar to those obtained under the stress scenarios reported here and are available upon request.
}

3.4.3. Stress scenario 3 (expected returns, volatilities and correlations)

In this case, we lower the conditional expected returns and in crease the conditional volatilities as in scenario 2 , but in addition we apply a haircut to the conditional correlations, i.e. $\widetilde{P}_{t} P_{t}+$ $\Delta_{P}$, where $\Delta_{P}$ is the haircut level for the correlations. In this case, $\operatorname{sVaR}_{t} \quad w^{\prime} \mu_{t}+\left(w^{\prime} \widetilde{D}_{t} \widetilde{P}_{t} \widetilde{D}_{t} w\right)^{1 / 2} q$. An important issue that arises when "stressing" the correlation matrix is that the resulting matrix $\widetilde{P}_{t}$ may not be positive definite. To circumvent this problem, we employ the approach proposed by Qi and Sun (2010), which is de signed to obtain the nearest positive definite correlation matrix in the context of the sVaR.

\subsubsection{Definition of the haircut parameters}

In order to obtain the stressed conditional moments for each of the stress scenarios defined above, it is necessary to define specific values for the haircut levels to be applied to expected re turns, volatilities, and correlations. Our choices for the haircut levels are similar to those considered in the stress testing exer cise conducted by the European regulatory authorities; see Com mittee of European Banking Supervisors (2010). Specifically, we set the haircut applied to the expected returns such that they be come equal to $20 \%$ (in annualized percentage points). Moreover, we set the haircut applied to the volatilities such that volatility for each individual asset doubles. Finally, we set the haircut applied to the correlations such that the correlations (which typically are positive) among each pair of assets doubles. Consid ering that some assets may already have correlations larger than 0.5 , we choose to 'cap' the correlations at 0.95 , i.e. set $\rho_{i j t}$ min (2 $\left.\rho_{i j t}, 0.95\right)$.

It is worth noting that, in the case of the futures portfolio, some currencies and commodities are often considered to be 'safe ha vens', so that during crisis periods their prices increase rather than decline. The same may also apply to (some) government bonds. By making this assumption, we may increase the expected returns of those assets, rather than lower them. We assume that gold, silver, Swiss Franc, and US government bonds are such 'safe havens' and that their expected returns are increased by $20 \%$ (in annualized percentage points). Accordingly, we assume that the correlations with the equity index futures (and other bonds, currencies and commodities) fall by half. For the 'non safe haven' bonds, curren cies, and commodities, we assume a decline in expected returns and an increase in correlation with the equity index futures using the same haircut levels discussed above.

\subsection{Implementation details}

We use a rolling window of $\tau=1000$ observations to estimate the parameters of the models that are used for generating the ex pected returns and the conditional covariance matrix. The follow ing stepwise procedure is then used to obtain optimal MCR portfolios:

1. Using the observations for $t=1, \ldots, \tau$, estimate the coefficients in the VAR (1) model (15) and in the model for the conditional covariance matrix.

2. Compute the expected return $\mu_{\tau+1}$ and the conditional covari ance matrix $H_{\tau+1}$, and their corresponding stressed counter parts, $\mu_{\tau+1}$ and $\widetilde{H}_{\tau+1}$, respectively, according to each of the stressed scenarios discussed in SubSection 3.4.

3. Use the last 250 observations up to observation $\tau$ to solve the optimization problem in (14) and obtain the optimal MCR port folio weights $w_{\tau}$ for day $\tau+1$.

4. Compute the portfolio return for day $\tau+1$ as $r_{p, \tau+1} \quad w_{\tau}^{\prime} R_{\tau+1}$, the portfolio $\operatorname{VaR}$ as $\operatorname{VaR}_{\tau+1} \quad w_{\tau}^{\prime} \mu_{\tau+1}+\left(w_{\tau}^{\prime} H_{\tau+1} w_{\tau}\right)^{1 / 2} q$, and the portfolio $\mathrm{sVaR}$ as $\mathrm{sVaR}_{\tau+1} \quad w_{\tau}^{\prime} \mu_{\tau+1}+\left(w_{\tau}^{\prime} \widetilde{H}_{\tau+1} w_{\tau}\right)^{1 / 2} q$. 
5. Move to the next window with observations $t=2, \ldots, \tau+1$ and repeat steps 14 until the end of the sample is reached.

After completing these steps, we have a total of $T \quad \tau$ out of sample observations for the portfolio return and one step ahead estimates of the portfolio VaR and sVaR, where $T$ denotes the sam ple size.

The following two points, related to our choice of models for the conditional covariance matrix, are useful to note. First, the Risk Metrics approach does not involve any unknown coefficients as we set $\lambda=0.94$. Second, the shrinkage estimator of Ledoit and Wolf (2003) assumes that the covariance matrix is constant during the estimation window. When the shrinkage estimator is computed using an estimation window up to observation $\tau$, we set the condi tional covariance matrix $H_{\tau+1}$ equal to the resulting estimate.

The performance of the MCR portfolios depends on a good choice of the parameter $\delta$ in (13), which controls the desired max imum number of VaR violations. In order to calibrate this parame ter, we use the following cross validation procedure. ${ }^{11}$ Using the parameter estimates in the models for the conditional mean and for the conditional covariance matrix obtained with the first window of $\tau=1000$ observations, we solve the MCR porfolio optimization problem in (14) for $t=250, \ldots, \tau \quad 1$ for a range of values of $\delta$. We then pick the value of $\delta$ that minimizes the average capital require ments such that for each observation the maximum number of VaR violations (over the most recent 250 days) is less than or equal to 9 , which is the upper bound for the yellow zone according to Table 1 . The selected value for $\delta$ is used to obtain the MCR portfolios for the remaining observations of the data set. This procedure is imple mented separately for each of the models for the conditional covari ance matrix discussed in Section 3.2 and also for each of the stress scenarios discussed in Section 3.4.

\subsection{Out of sample evaluation}

Most important for the evaluation of the MCR portfolios are the characteristics of the daily capital requirement (DCR) and the num ber of VaR violations, both in an absolute sense and compared to the benchmark portfolios. For each specification of the covariance matrix (RM, DCC, and LW), we consider the mean daily capital requirement ("mean DCR"), the average number of VaR violations ("Mean Hit"), the maximum number of VaR violations ("Max Hit"), and the fraction of days for which the number of VaR viola tions is either in the green zone (i.e. below 5 ) or in the red zone (i.e. above 9). The statistics concerning the VaR exceedances are based on rolling periods of 250 out of sample observations, which is the time period established by the Basel II accord to evaluate the finan cial institutions' VaR disclosures.

We also examine the portfolios' performance in terms of the average gross return $(\hat{\mu})$, standard deviation of returns $(\hat{\sigma})$, Sharpe ratio (SR) and turnover. These statistics are computed as follows:

$$
\begin{aligned}
& \hat{\mu} \quad \frac{1}{T \tau} \sum_{t}^{T} w_{t}^{1} R_{t+1} \\
& \hat{\sigma} \quad \sqrt{\frac{1}{T \tau 1} \sum_{t} \tau}{ }_{t}^{T}\left(w_{t}^{\prime} R_{t+1} \quad \hat{\mu}\right)^{2} \\
& \text { SR } \frac{\hat{\mu}}{\hat{\sigma}} \\
& \text { Turnover } \frac{1}{T \tau} 1 \sum_{t}^{T} \sum_{j}^{1} \sum_{1}^{N}\left(\left|w_{j, t+1} \quad w_{j, t^{+}}\right|\right) \text {, }
\end{aligned}
$$

where $w_{j, t^{+}}$is the portfolio weight in asset $j$ at time $t+1$ but before rebalancing and $w_{j, t+1}$ is the desired portfolio weight in asset $j$ at

\footnotetext{
11 See Efron and Gong (1983) for a detailed explanation and DeMiguel et al. (2009a) for an application in the context of portfolio optimization.
}

time $t+1$. As pointed out by DeMiguel et al. (2009b), turnover as defined above can be interpreted as the average fraction of wealth traded in each period. ${ }^{12}$

To measure the impact of transaction costs on the performance of the different portfolios, we consider the average portfolio returns net of transaction costs, $\hat{\mu}_{T C}$, defined as

$\left.\hat{\mu}_{T C} \quad \frac{1}{T \tau} \sum_{t}^{T} \tau\left[\begin{array}{lll}1+w_{t}^{\prime} R_{t+1}\end{array}\right)\left(\begin{array}{ccc}1 & c \sum_{j 1}^{N} \mid w_{j, t+1} & w_{j, t+} \mid\end{array}\right) \quad 1\right]$

where $c$ is the fee to be paid for each transaction. Instead of assum ing an arbitrary value of $c$, we report the value of the breakeven transaction cost (Han, 2006). In other words, we report the value of $c$ that makes the average portfolio return net of transaction costs equal to zero. Note that, when comparing two alternative portfolio strategies, the one with a higher breakeven cost is to be preferred.

To test the hypothesis that the capital requirement levels, the number of VaR exceedances, and the Sharpe ratios obtained with the MCR portfolios and with the benchmark portfolios are equal, we follow DeMiguel et al. (2009a) and use the stationary bootstrap of Politis and Romano (1994) with $B=1000$ bootstrap resamples and expected block length $b=5 .^{13}$ The resulting bootstrap $p$ values are obtained using the methodology suggested in Ledoit and Wolf (2008, Remark 3.2).

\subsection{Results}

\subsubsection{Original Basel II capital requirements}

We first consider the MCR portfolios in (14) using as objective function the original Basel II capital requirement formula in (1), which does not involve the stressed VaR. For this reason, we do not include the Min sVaR portfolio in this analysis, but limit the comparison with the Min VaR and $1 / N$ portfolios as benchmarks. Table 2 reports the daily capital requirements, the number of VaR violations, and the performance of each portfolio strategy in terms of average gross returns, standard deviation of returns, Sharpe ratio, turnover, and breakeven transaction costs. Returns, standard deviation and Sharpe ratios are annualized. Returns and volatilities are reported in percentages and breakeven transaction costs in terms of basis points (bp).

The results for the mixed futures portfolio in Panel A of Table 2 indicate that, irrespective of the model used for the conditional covariance matrix, the MCR portfolio achieves its purpose in the sense that it leads to lower mean capital requirements than the Min VaR and $1 / N$ portfolios. The lowest mean DCR of $1.10 \%$ is achieved when the Risk Metrics model is used. Reassuringly, these lower capital requirements do not come at the expense of a larger number of VaR violations. In fact, the mean number of VaR excee dances (Mean Hit) for the MCR portfolios is always lower than for the two benchmarks, except when the DCC model is used as the Min VaR portfolio performs slightly better in this respect. For all covariance matrix specifications, the mean number of VaR excee dances for the MCR portfolios is below 4, which corresponds with the upper bound of the green zone in Table 1. Therefore, the per centage of days spent in the green zone (such that the penalty fac tor $k$ in (1) is equal to zero) is quite satisfactory, and considerably higher than for the two benchmark portfolios. Except for the $1 / N$ portfolio when the LW model is used, none of portfolio policies suf fer from more than nine VaR violations during the previous 250

\footnotetext{
12 Note that, in the case of an equally weighted (or $1 / N$ ) portfolio composition, we have $w_{j, t}=w_{j, t+1}=1 / N$, but $w_{j, t^{+}}$may be different due to changes in asset prices between $t$ and $t+1$.

${ }^{13}$ We performed extensive robustness checks regarding the choice of the block length, using a range of values for $b$ between 5 and 250. Regardless of the block length, the test results for the differences in capital requirements, VaR exceedances and Sharpe ratios are similar to those reported here.
} 
Table 2

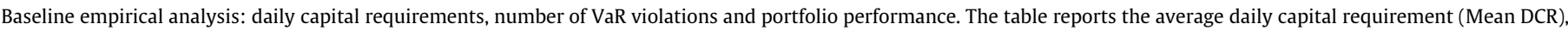

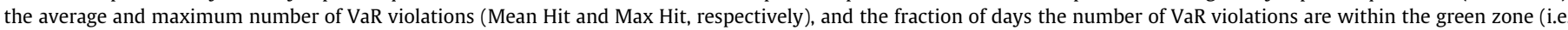

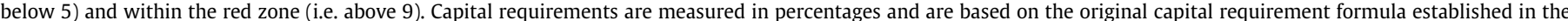

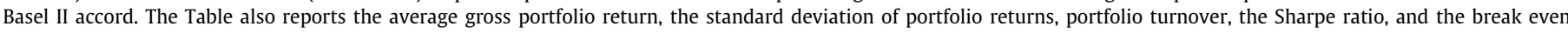

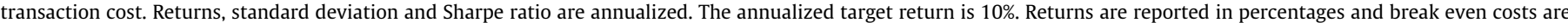

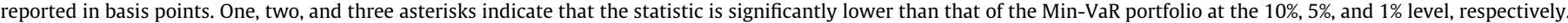
All figures are based on subsequent (rolling) periods of 250 out-of-sample observations.

\begin{tabular}{|c|c|c|c|c|c|c|c|c|c|c|}
\hline & \multicolumn{5}{|c|}{ Capital requirements } & \multicolumn{5}{|c|}{ Portfolio performance } \\
\hline & Mean DCR (\%) & Mean Hit & $\begin{array}{l}\text { Max } \\
\text { Hit }\end{array}$ & $\begin{array}{l}\% \text { of days in } \\
\text { red zone }\end{array}$ & $\begin{array}{l}\% \text { of days in } \\
\text { green zone }\end{array}$ & $\begin{array}{l}\text { Gross } \\
\text { returns (\%) }\end{array}$ & Std. deviation & $\begin{array}{l}\text { Sharpe } \\
\text { ratio }\end{array}$ & Turnover & $\begin{array}{l}\text { Break even } \\
\text { cost (b.p.) }\end{array}$ \\
\hline \multicolumn{11}{|c|}{ Panel A: mixed futures portfolio } \\
\hline MCR & 1.22 & 3.72 & 8 & 0.00 & 72.78 & 4.55 & 3.49 & 1.30 & 0.46 & 3.87 \\
\hline Min-VaR & 1.23 & 2.96 & 6 & 0.00 & 76.27 & 4.40 & 3.80 & 1.16 & 0.69 & 2.50 \\
\hline $1 / N$ & 2.84 & 4.45 & 8 & 0.00 & 56.36 & 11.62 & 8.76 & 1.33 & 0.01 & 715.00 \\
\hline \multicolumn{11}{|c|}{ Covariance model: Risk Metrics } \\
\hline MCR & $1.10^{* * *}$ & $3.65^{* * *}$ & 6 & 0.00 & 91.00 & 4.31 & 3.46 & 1.25 & 0.49 & 3.49 \\
\hline Min-VaR & 1.20 & 6.16 & 8 & 0.00 & 34.96 & 5.17 & 3.95 & 1.31 & 0.79 & 2.56 \\
\hline $1 / N$ & 3.10 & 4.45 & 8 & 0.00 & 64.19 & 11.62 & 8.76 & 1.33 & 0.01 & 715.00 \\
\hline \multicolumn{11}{|c|}{ Covariance model: Ledoit-Wolf } \\
\hline MCR & 1.41 & 1.18 & 6 & 0.00 & 96.08 & 3.47 & 3.82 & 0.91 & 0.44 & 3.08 \\
\hline Min-VaR & 1.49 & 1.52 & 6 & 0.00 & 96.08 & 0.34 & 4.99 & 0.07 & 0.68 & 0.20 \\
\hline $1 / N$ & 3.07 & 7.00 & 16 & 19.49 & 46.61 & 11.62 & 8.76 & 1.33 & 0.01 & 715.00 \\
\hline \multicolumn{11}{|c|}{ Panel B: US industry portfolios } \\
\hline \multicolumn{11}{|c|}{ Covariance model: DCC } \\
\hline MCR & 6.81 & $2.67^{* * *}$ & 5 & 0.00 & 98.39 & 23.87 & 20.20 & $1.18^{*}$ & 0.34 & 25.58 \\
\hline Min-VaR & 3.52 & 10.53 & 18 & 34.49 & 32.18 & 5.12 & 10.80 & 0.47 & 0.99 & 2.03 \\
\hline $1 / N$ & 5.00 & 8.34 & 15 & 22.61 & 37.37 & 6.73 & 14.30 & 0.47 & 0.01 & 503.80 \\
\hline \multicolumn{11}{|c|}{ Covariance model: Risk Metrics } \\
\hline MCR & 6.63 & $4.44^{* * *}$ & 7 & 0.00 & 65.40 & 9.84 & 19.05 & 0.52 & 0.54 & 7.03 \\
\hline Min-VaR & 3.41 & 11.09 & 16 & 48.67 & 28.84 & 7.11 & 10.50 & 0.68 & 1.04 & 2.66 \\
\hline $1 / N$ & 5.09 & 6.12 & 10 & 5.31 & 46.14 & 6.73 & 14.30 & 0.47 & 0.01 & 503.80 \\
\hline \multicolumn{11}{|c|}{ Covariance model: Ledoit-Wolf } \\
\hline MCR & 6.81 & $3.50^{* * *}$ & 10 & 6.57 & 78.43 & 26.91 & 18.80 & $1.43^{* * *}$ & 0.37 & 26.33 \\
\hline Min-VaR & 3.60 & 10.66 & 25 & 28.84 & 42.21 & 3.68 & 11.31 & 0.33 & 0.80 & 1.82 \\
\hline $1 / N$ & 4.58 & 8.44 & 24 & 22.61 & 63.32 & 6.73 & 14.30 & 0.47 & 0.01 & 503.80 \\
\hline
\end{tabular}

business days at any point in time, such that the red zone is never entered. In terms of the traditional performance criteria, the aver age gross returns of the MCR portfolios vary between $3.5 \%$ and $4.5 \%$, falling short of the annualized target return of $10 \%$. The same holds for the Min VaR portfolio. The $1 / N$ performs better in terms of mean returns, but this is accompanied by a standard deviation that is twice as large as for the MCR and Min VaR portfolios. Con sequently, the risk adjusted performance measured by the Sharpe ratio are similar across the different portfolios and are never signif icantly different. Turnover is substantially lower for the MCR port folios compared to the Min VaR portfolios, such that the breakeven transaction costs are considerably higher for the MCR portfolios. As expected, by far the lowest turnover is achieved by the $1 / \mathrm{N}$ portfo lio, since changes in portfolios compositions are solely due to changes in asset prices. In fact, for this data set, the best perfor mance in terms of returns, turnover and breakeven transaction costs is achieved by the $1 / N$ portfolio. This result corroborates pre vious findings in the literature, such as DeMiguel et al. (2009b) regarding the outperformance of the $1 / N$ portfolio vis à vis more sophisticated portfolio strategies.

The results for the US industry portfolios in panel B of Table 2 also illustrate the trade off between capital requirement levels and the number of VaR violations mentioned above. At first glance, one could argue that the MCR portfolios do not perform well, in the sense that they render a higher mean DCR than both benchmark portfolios. This conclusion is, however, misleading: The lower cap ital charges for the benchmark portfolios come at the expense of a very high number of VaR violations, leading to a rather substantial fraction of days spent in the red zone. As discussed before, this is highly undesirable due to potential damaging effects on the banks' reputation regarding their risk management systems. For instance, using the DCC model for the Min VaR portfolio delivers an average and maximum number of $\mathrm{VaR}$ violations equal to 10.53 and 18 , respectively, leading to a total of $34 \%$ of days in the red zone. On the other hand, for the MCR strategy, the corresponding numbers are 2.67 and 5 , altogether avoiding the red zone during the entire out of sample period. In terms of portfolio performance, the re sults are largely in favor of the MCR strategy. The MCR portfolios deliver average gross returns substantially higher than the Min VaR and $1 / N$ portfolios for all covariance matrix specifications, and also higher than the annualized target return of $10 \%$ when the DCC model or LW approach are used. In those cases, even though volatility of the MCR portfolios is higher as well, the Sharpe ratio of the MCR portfolios is significantly higher than those of the two benchmarks. Again we find that the turnover for the MCR port folio is lower than for the Min VaR portfolio in all situations. The higher gross returns combined with the lower turnover lead to breakeven transaction costs for the MCR portfolio that are much higher than those for the Min VaR portfolio.

\subsubsection{New Basel II capital requirements}

Next we examine the MCR portfolio strategy when the amend ments to the Basel II accord involving the sVaR are taken into ac count. Tables 35 report the results for capital requirements and performance measures for all portfolio policies under stress sce narios 13 , respectively. The characteristics of the stress scenarios are discussed in Section 3.4. Capital requirements are computed according to the new Basel II specification in (2), which does re quire the estimation of the sVaR. For each stress scenario, we ob tain the sVaR estimates using the haircut values discussed in 
Table 3

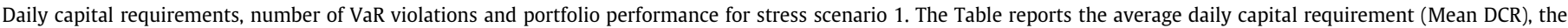

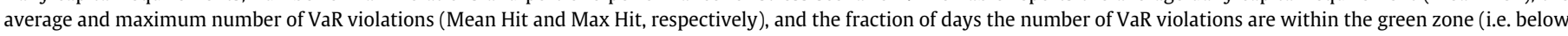

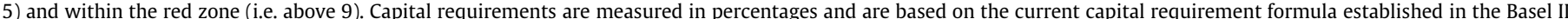

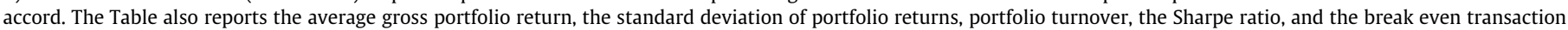

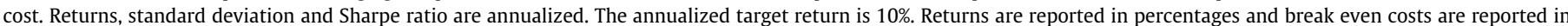

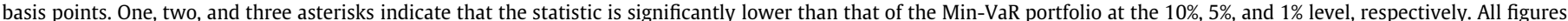
are based on subsequent (rolling) periods of 250 out-of-sample observations.

\begin{tabular}{|c|c|c|c|c|c|c|c|c|c|c|}
\hline & \multicolumn{5}{|c|}{ Capital requirements } & \multicolumn{5}{|c|}{ Portfolio Performance } \\
\hline & Mean DCR (\%) & Mean Hit & Max Hit & $\begin{array}{l}\% \text { of days in } \\
\text { red zone }\end{array}$ & $\begin{array}{l}\% \text { of days in } \\
\text { green zone }\end{array}$ & $\begin{array}{l}\text { Gross } \\
\text { returns (\%) }\end{array}$ & Std. deviation & Sharpe ratio & Turnover & $\begin{array}{l}\text { Break even } \\
\text { cost (b.p.) }\end{array}$ \\
\hline \multicolumn{11}{|c|}{ Panel A: mixed futures portfolio } \\
\hline \multicolumn{11}{|c|}{ Covariance model: DCC } \\
\hline MCR & 2.45 & 3.72 & 8 & 0.00 & 72.78 & 4.46 & 3.50 & 1.28 & 0.45 & 3.87 \\
\hline Min-VaR & 2.43 & 2.42 & 5 & 0.00 & 92.16 & 4.40 & 3.80 & 1.16 & 0.69 & 2.50 \\
\hline Min-sVaR & 2.46 & 3.32 & 7 & 0.00 & 75.95 & 3.62 & 3.88 & 0.93 & 0.66 & 2.18 \\
\hline $1 / N$ & 5.68 & 4.45 & 8 & 0.00 & 56.36 & 11.62 & 8.76 & 1.33 & 0.01 & 715.00 \\
\hline \multicolumn{11}{|c|}{ Covariance model: Risk Metrics } \\
\hline MCR & $2.19^{* * *}$ & $4.45^{* * *}$ & 10 & 1.27 & 66.74 & 4.67 & 3.75 & 1.25 & 0.43 & 4.22 \\
\hline Min-VaR & 2.39 & 5.67 & 9 & 0.00 & 37.71 & 5.17 & 3.95 & 1.31 & 0.79 & 2.56 \\
\hline Min-sVaR & 2.75 & $3.57^{* * *}$ & 5 & 0.00 & 88.03 & 2.91 & 7.29 & 0.40 & 0.99 & 1.17 \\
\hline $1 / N$ & 4.08 & $4.45^{* * *}$ & 8 & 0.00 & 64.19 & 11.62 & 8.76 & 1.33 & 0.01 & 715.00 \\
\hline \multicolumn{11}{|c|}{ Panel B: US industry portfolios } \\
\hline \multicolumn{11}{|c|}{ Covariance model: DCC } \\
\hline MCR & 13.12 & $2.67^{* * * *}$ & 5 & 0.00 & 98.39 & 25.95 & 19.39 & $1.34^{* *}$ & 0.33 & 28.63 \\
\hline Min-VaR & 6.93 & 7.28 & 14 & 26.87 & 58.48 & 5.12 & 10.80 & 0.47 & 0.99 & 2.03 \\
\hline Min-sVaR & 7.27 & 10.53 & 18 & 34.49 & 32.18 & 4.36 & 11.13 & 0.39 & 1.01 & 1.69 \\
\hline $1 / N$ & 10.00 & 8.34 & 15 & 22.61 & 37.37 & 6.73 & 14.30 & 0.47 & 0.01 & 503.80 \\
\hline \multicolumn{11}{|c|}{ Covariance model: Risk Metrics } \\
\hline MCR & 10.35 & $2.94^{* * *}$ & 6 & 0.00 & 97.12 & 13.84 & 19.88 & 0.70 & 0.63 & 8.28 \\
\hline Min-VaR & 6.03 & 9.29 & 14 & 31.49 & 28.84 & 7.11 & 10.50 & 0.68 & 1.04 & 2.66 \\
\hline Min-sVaR & 5.23 & $7.03^{* * * *}$ & 11 & 9.11 & 35.18 & 8.38 & 13.53 & 0.62 & 0.94 & 3.45 \\
\hline $1 / N$ & $5.78^{* * *}$ & $6.12^{* * *}$ & 10 & 5.31 & 46.14 & 6.73 & 14.30 & 0.47 & 0.01 & 503.80 \\
\hline
\end{tabular}

Section 3.4. The MCR portfolios are obtained by solving the optimi zation problem in (14). We now also include the Min sVaR as a third benchmark portfolio, in addition to the Min VaR and $1 / N$ portfolios. Finally, in this case, we do not consider the LW model for the covariances because it is not clear how to decompose the LW covariance matrix into standard deviations and correlations in order to stress them.

The results for the mixed futures portfolio reported in Panel A of Tables 35 are in line with the analysis based on the original Basel II capital requirement formulation as discussed above. When the Risk Metrics model is used, the MCR strategy delivers lower aver age capital requirements in comparison to all benchmark strate gies and across all stress scenarios. Compared to the baseline analysis, the mean DCR obviously increases substantially due to the extra term involving the sVaR in (2). The mean number of VaR exceedances declines accordingly, such that most of the time the number of violations during the past 250 business days does not exceed 4 . The MCR portfolio based on RiskMetrics forecasts of the covariances experiences a short period with 10 historic VaR violations, such that during $1.3 \%$ of the out of sample period the red zone is entered. We also observe that all portfolio strategies deliver similar Sharpe ratios in all stress scenarios. ${ }^{14}$ However, the MCR portfolios systematically achieve lower turnover than the Min VaR and Min sVaR portfolios, such that the breakeven transaction costs are considerably higher for the MCR portfolios in all specifica tions and across all stress scenarios.

\footnotetext{
14 Note that the performance of the Min-VaR and the $1 / N$ strategies in terms of gross returns, Sharpe ratio and transaction costs is the same across all stress scenarios. This is due to the fact that the optimal portfolio weights in these strategies are not determined using stressed conditional moments. On the other hand, the optimal portfolio weights under the MCR and Min-sVaR strategies do consider stress conditional moments in their formulation. Therefore, their performance varies across stress scenarios.
}

The results for the US industry portfolios reported in Panel B of Tables 35 are even more favorable to the MCR strategy, where again it achieves a much better balance in terms of capital require ment levels and the number of VaR violations. In all stress scenar ios, although the average DCR for the MCR strategy is higher in comparison to the benchmark policies, the number of VaR excee dances under the MCR strategy is the lowest and never exceeds the upper bound of the yellow zone. For instance, when imple menting the DCC model for the conditional covariance matrices, the average number of VaR violations are 2.67, 3.09, and 2.78, in scenarios 13 , respectively. The same numbers for the Min sVaR policy are $10.53,9.66$, and 8.50 which end up leading to a high frac tion of days in the red zone. Furthermore, the performance of the MCR strategy in terms of average gross returns and Sharpe ratio is consistently better in comparison to the benchmark strategies. Finally, we observe that in all stress scenarios the MCR strategy delivers lower turnover and considerably higher breakeven trans action costs in comparison to the Min VaR and Min sVaR policies.

To further illustrate the results, Figs. 13 plot, for scenarios 13 respectively, the evolution of the number of VaR violations over the previous trading year and the daily capital charge for the MCR (dashed line), Min VaR (solid blue line), and Min sVaR (solid cyan line) portfolios when the DCC specification is used for the condi tional covariance matrix. We also plot a horizontal line indicating the threshold value for the red zone ( $9 \mathrm{VaR}$ exceedances in the pre vious 250 trading days). These graphs show that for the mixed fu tures portfolio the DCR and the number of VaR violations for the three portfolio policies are rather similar. For the US industry port folios, however, we observe that while the DCR is higher for the MCR portfolios, the number of VaR violations are much lower and

equally important remain below the red zone threshold during the complete out of sample period. The Min VaR and Min sVaR portfolios, in contrast, deliver a number of VaR violations that are deep in the red zone during a large part of the out of sample period. 
Table 4

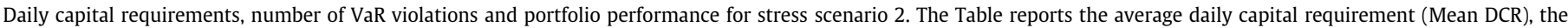

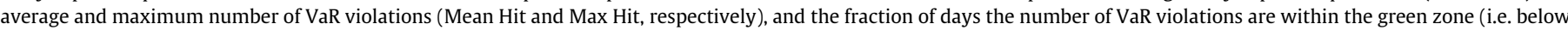

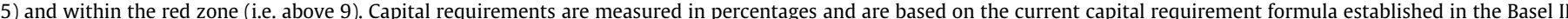

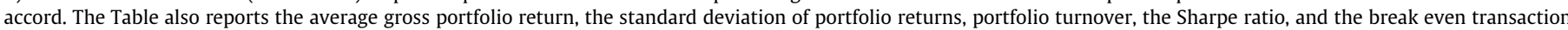

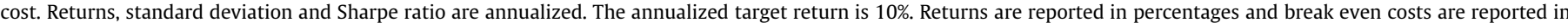

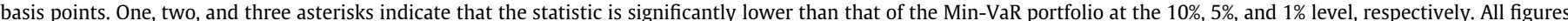
are based on subsequent (rolling) periods of 250 out-of-sample observations.

\begin{tabular}{|c|c|c|c|c|c|c|c|c|c|c|}
\hline & \multicolumn{5}{|c|}{ Capital requirements } & \multicolumn{5}{|c|}{ Portfolio performance } \\
\hline & Mean DCR (\%) & Mean Hit & Max Hit & $\begin{array}{l}\% \text { of days } \\
\text { in red zone }\end{array}$ & $\begin{array}{l}\% \text { of days } \\
\text { in green zone }\end{array}$ & Gross returns (\%) & Std. deviation & Sharpe ratio & Turnover & $\begin{array}{l}\text { Break even } \\
\text { cost (b.p.) }\end{array}$ \\
\hline \multicolumn{11}{|c|}{ Panel A: mixed futures portfolio } \\
\hline MCR & $2.90^{* * *}$ & 3.09 & 5 & 0.00 & 91.84 & 4.10 & 3.84 & 1.07 & 0.46 & 3.54 \\
\hline Min-VaR & 3.28 & 2.42 & 5 & 0.00 & 92.16 & 4.40 & 3.80 & 1.16 & 0.69 & 2.50 \\
\hline Min-sVaR & $2.85^{* * *}$ & $2.37^{*}$ & 6 & 0.00 & 91.31 & 4.10 & 4.37 & 0.94 & 0.56 & 2.89 \\
\hline $1 / N$ & 8.19 & 4.45 & 8 & 0.00 & 56.36 & 11.62 & 8.76 & 1.33 & 0.01 & 715.00 \\
\hline \multicolumn{11}{|c|}{ Covariance model: Risk Metrics } \\
\hline MCR & $2.57^{* * *}$ & $4.39^{* * *}$ & 7 & 0.00 & 59.43 & 4.40 & 3.92 & 1.12 & 0.43 & 4.01 \\
\hline Min-VaR & 3.24 & 5.67 & 9 & 0.00 & 37.71 & 5.17 & 3.95 & 1.31 & 0.79 & 2.56 \\
\hline Min-sVaR & $2.62^{*}$ & $3.30^{* * *}$ & 7 & 0.00 & 82.63 & 4.22 & 6.24 & 0.68 & 0.75 & 2.21 \\
\hline $1 / N$ & 4.94 & $4.45^{* * *}$ & 8 & 0.00 & 64.19 & 11.62 & 8.76 & 1.33 & 0.01 & 715.00 \\
\hline \multicolumn{11}{|c|}{ Panel B: US industry portfolios } \\
\hline MCR & 20.49 & $3.32^{* * *}$ & 6 & 0.00 & 83.28 & 18.81 & 19.39 & 0.97 & 0.37 & 19.07 \\
\hline Min-VaR & 10.40 & 7.28 & 14 & 26.87 & 58.48 & 5.12 & 10.80 & 0.47 & 0.99 & 2.03 \\
\hline Min-sVaR & 10.46 & 9.66 & 17 & 28.03 & 32.18 & 4.50 & 10.63 & 0.42 & 0.72 & 2.44 \\
\hline $1 / N$ & 15.00 & 8.34 & 15 & 22.61 & 37.37 & 6.73 & 14.30 & 0.47 & 0.01 & 503.80 \\
\hline \multicolumn{11}{|c|}{ Covariance model: Risk Metrics } \\
\hline MCR & 13.97 & $2.30^{* * *}$ & 6 & 0.00 & 97.12 & 13.60 & 20.05 & 0.68 & 0.63 & 8.16 \\
\hline Min-VaR & 8.50 & 9.29 & 14 & 31.49 & 28.84 & 7.11 & 10.50 & 0.68 & 1.04 & 2.66 \\
\hline Min-sVaR & $5.64^{* * *}$ & $7.03^{* * *}$ & 11 & 9.11 & 35.18 & 8.05 & 13.39 & 0.60 & 0.73 & 4.27 \\
\hline $1 / N$ & $6.46^{* * *}$ & $6.12^{* * *}$ & 10 & 5.31 & 46.14 & 6.73 & 14.30 & 0.47 & 0.01 & 503.80 \\
\hline
\end{tabular}

Table 5

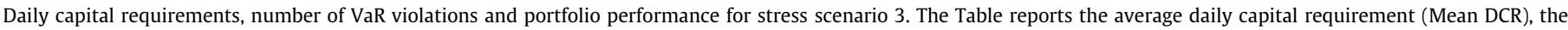

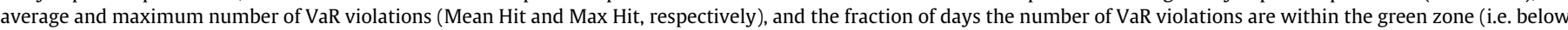

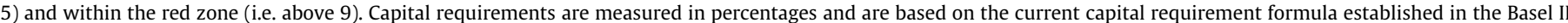

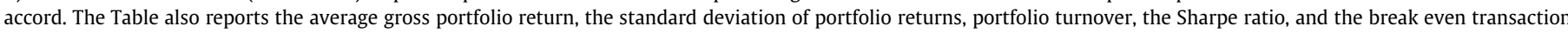

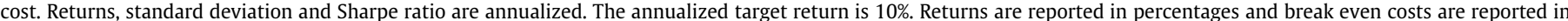

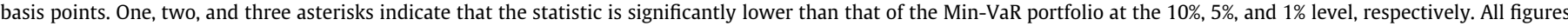
are based on subsequent (rolling) periods of 250 out-of-sample observations.

\begin{tabular}{|c|c|c|c|c|c|c|c|c|c|c|}
\hline & \multicolumn{5}{|c|}{ Capital requirements } & \multicolumn{5}{|c|}{ Portfolio performance } \\
\hline & Mean DCR (\%) & Mean Hit & Max Hit & $\begin{array}{l}\% \text { of days } \\
\text { in red zone }\end{array}$ & $\begin{array}{l}\% \text { of days in } \\
\text { green zone }\end{array}$ & $\begin{array}{l}\text { Gross returns } \\
(\%)\end{array}$ & Std. deviation & Sharpe ratio & Turnover & $\begin{array}{l}\text { Break even } \\
\text { cost (b.p.) }\end{array}$ \\
\hline \multicolumn{11}{|c|}{ Panel A: mixed futures portfolio } \\
\hline \multicolumn{11}{|c|}{ Covariance model: DCC } \\
\hline MCR & $3.02^{* * *}$ & 2.78 & 5 & 0.00 & 91.95 & 3.99 & 3.98 & 1.00 & 0.49 & 3.19 \\
\hline Min-VaR & 3.55 & 2.42 & 5 & 0.00 & 92.16 & 4.40 & 3.80 & 1.16 & 0.69 & 2.50 \\
\hline Min-sVaR & $2.92^{* * *}$ & $2.18^{* * *}$ & 6 & 0.00 & 98.52 & 3.86 & 4.67 & 0.83 & 0.58 & 2.61 \\
\hline $1 / N$ & 9.67 & 4.45 & 8 & 0.00 & 56.36 & 11.62 & 8.76 & 1.33 & 0.01 & 715.00 \\
\hline \multicolumn{11}{|c|}{ Covariance model: Risk Metrics } \\
\hline MCR & $2.50^{* * *}$ & $4.00^{* * *}$ & 7 & 0.00 & 70.44 & 4.46 & 4.01 & 1.11 & 0.45 & 3.91 \\
\hline Min-VaR & 3.21 & 5.67 & 9 & 0.00 & 37.71 & 5.17 & 3.95 & 1.31 & 0.79 & 2.56 \\
\hline Min-sVaR & 3.41 & $3.65^{* * *}$ & 6 & 0.00 & 75.64 & 7.73 & 8.95 & 0.86 & 1.18 & 2.54 \\
\hline $1 / N$ & 4.77 & $4.45^{* * *}$ & 8 & 0.00 & 64.19 & 11.62 & 8.76 & 1.33 & 0.01 & 715.00 \\
\hline \multicolumn{11}{|c|}{ Panel B: US industry portfolios } \\
\hline \multicolumn{11}{|c|}{ Covariance model: DCC } \\
\hline MCR & 21.31 & $2.18^{* * *}$ & 5 & 0.00 & 98.15 & 18.78 & 20.62 & 0.91 & 0.38 & 18.50 \\
\hline Min-VaR & 11.82 & 7.28 & 14 & 26.87 & 58.48 & 5.12 & 10.80 & 0.47 & 0.99 & 2.03 \\
\hline Min-sVaR & $11.13^{* *}$ & 8.50 & 17 & 28.37 & 41.64 & 0.46 & 10.62 & 0.04 & 0.71 & 0.26 \\
\hline $1 / N$ & 17.60 & 8.34 & 15 & 22.61 & 37.37 & 6.73 & 14.30 & 0.47 & 0.01 & 503.80 \\
\hline \multicolumn{11}{|c|}{ Covariance model: Risk Metrics } \\
\hline MCR & 13.63 & $2.89^{* * *}$ & 5 & 0.00 & 95.16 & 15.14 & 20.02 & 0.76 & 0.64 & 8.89 \\
\hline Min-VaR & 8.44 & 9.29 & 14 & 31.49 & 28.84 & 7.11 & 10.50 & 0.68 & 1.04 & 2.66 \\
\hline Min-sVaR & $6.02^{* * *}$ & $7.92^{*}$ & 14 & 25.72 & 39.91 & 9.17 & 14.42 & 0.64 & 1.41 & 2.52 \\
\hline $1 / N$ & $6.37^{* * *}$ & $6.12^{* * *}$ & 10 & 5.31 & 46.14 & 6.73 & 14.30 & 0.47 & 0.01 & 503.80 \\
\hline
\end{tabular}

Two interesting results emerge from the analysis based on stress scenarios. First, we observe that as we move from scenarios
1 to 3 the average DCR tends to increase for all portfolio policies. This result is expected since the 'stress level' increases as we move 

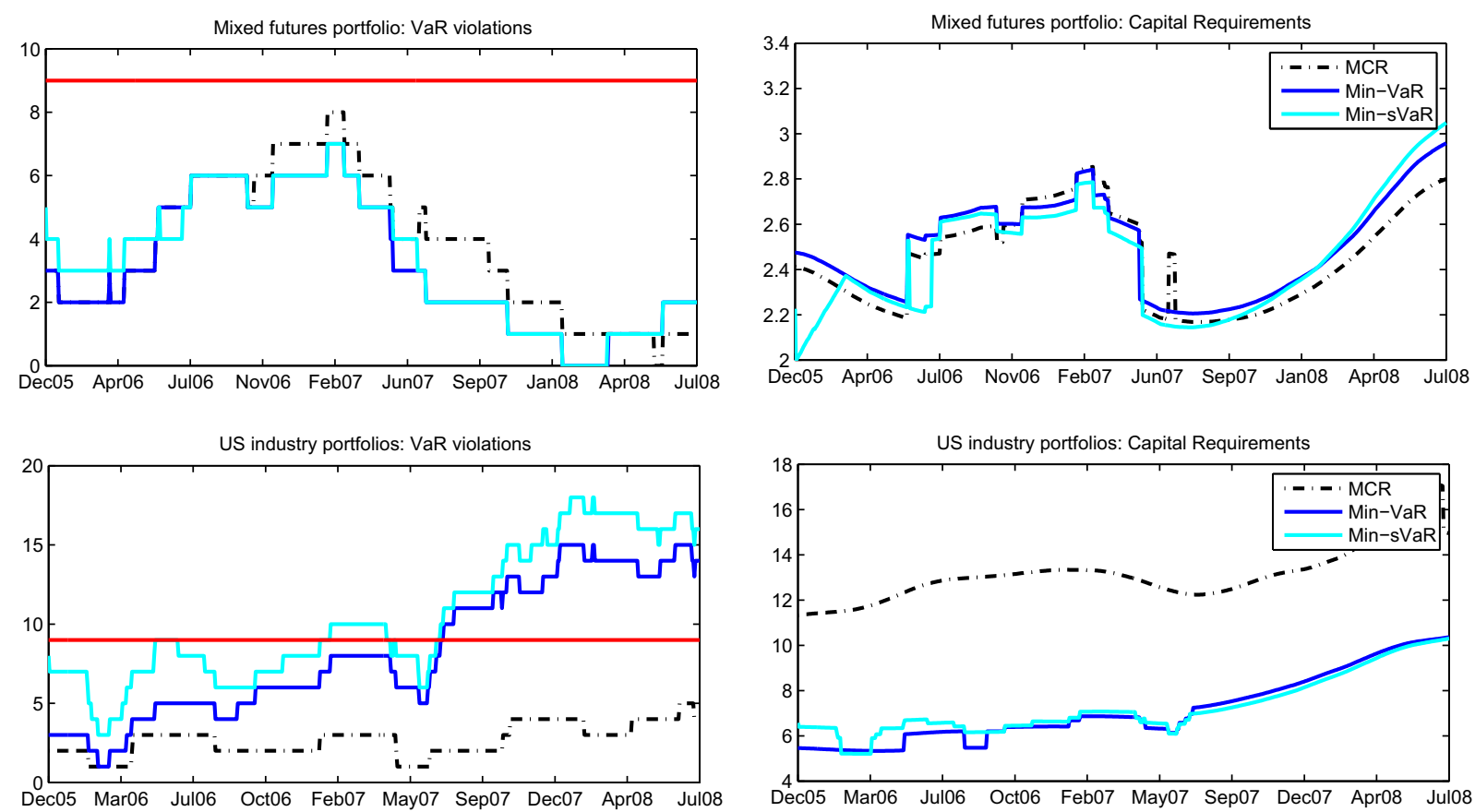

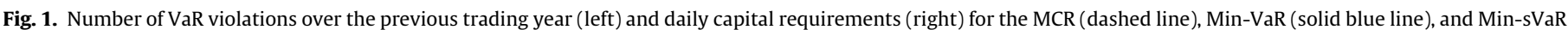

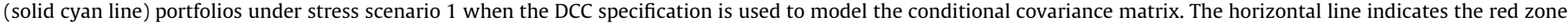

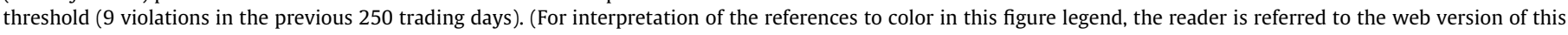
article.)
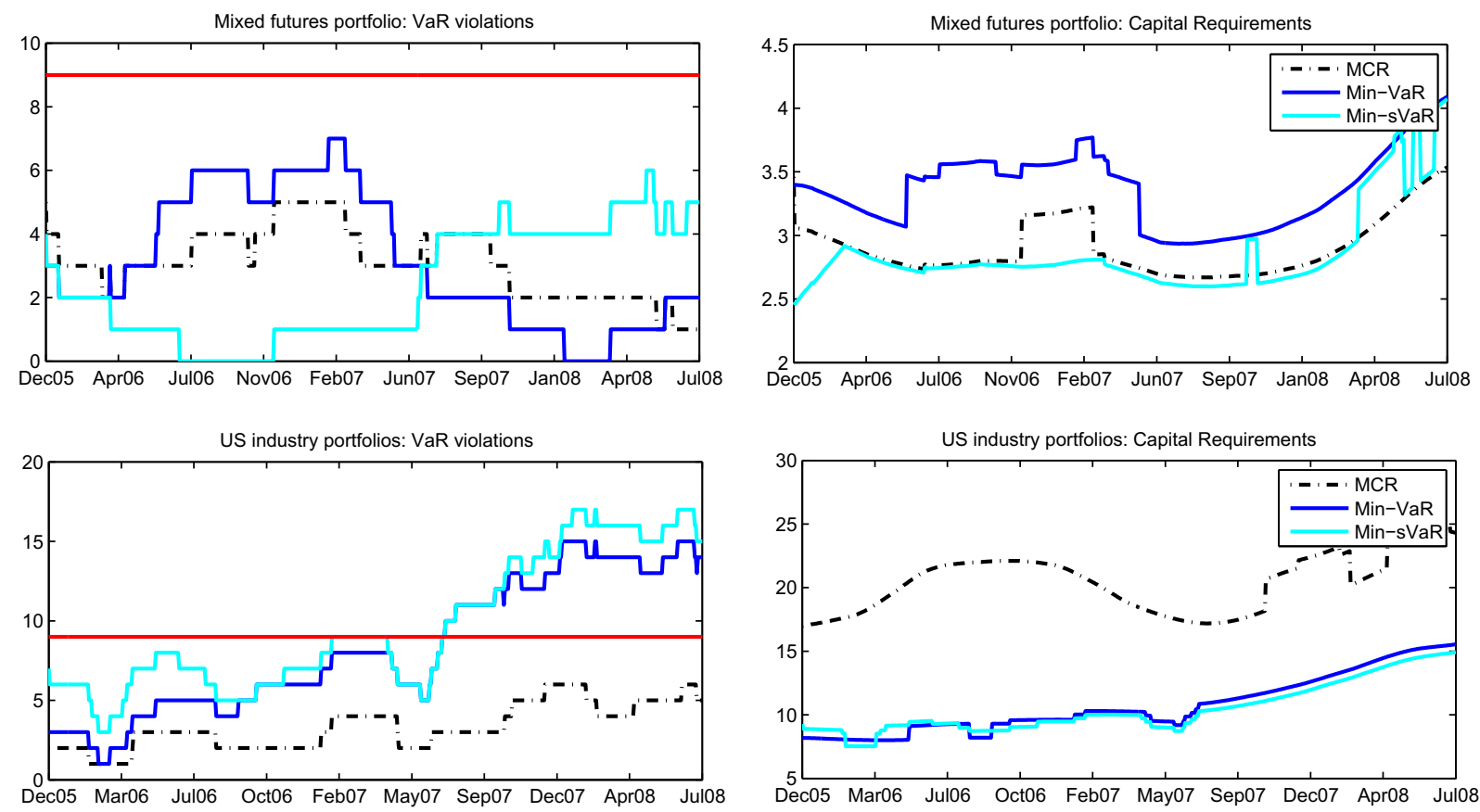

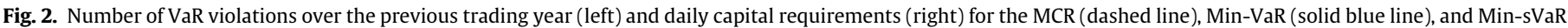

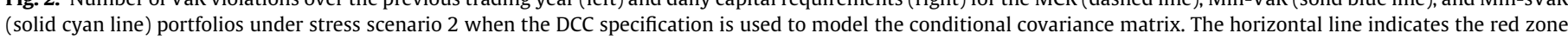

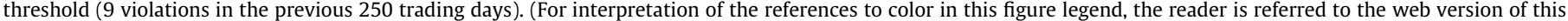
article.)

from scenarios 1 to 3 . Recall that in scenario 1 we only stress ex pected returns, while in scenario 3 we stress expected returns, vol atilities and correlations. Consequently, the DCR levels tend to increase. For instance, when the DCC model is used, the average DCR for the MCR strategy under stress scenarios 13 are $2.45 \%$, $2.90 \%$, and $3.02 \%$, respectively.
Second, previous studies found that the static $1 / N$ portfolio out performs several sophisticated dynamic portfolio strategies in terms of risk adjusted performance and transaction costs. While we corroborate this finding, we also note that the performance of the $1 / N$ portfolio may be not so promising from the risk manage ment point of view. For instance, in the mixed futures portfolio, 

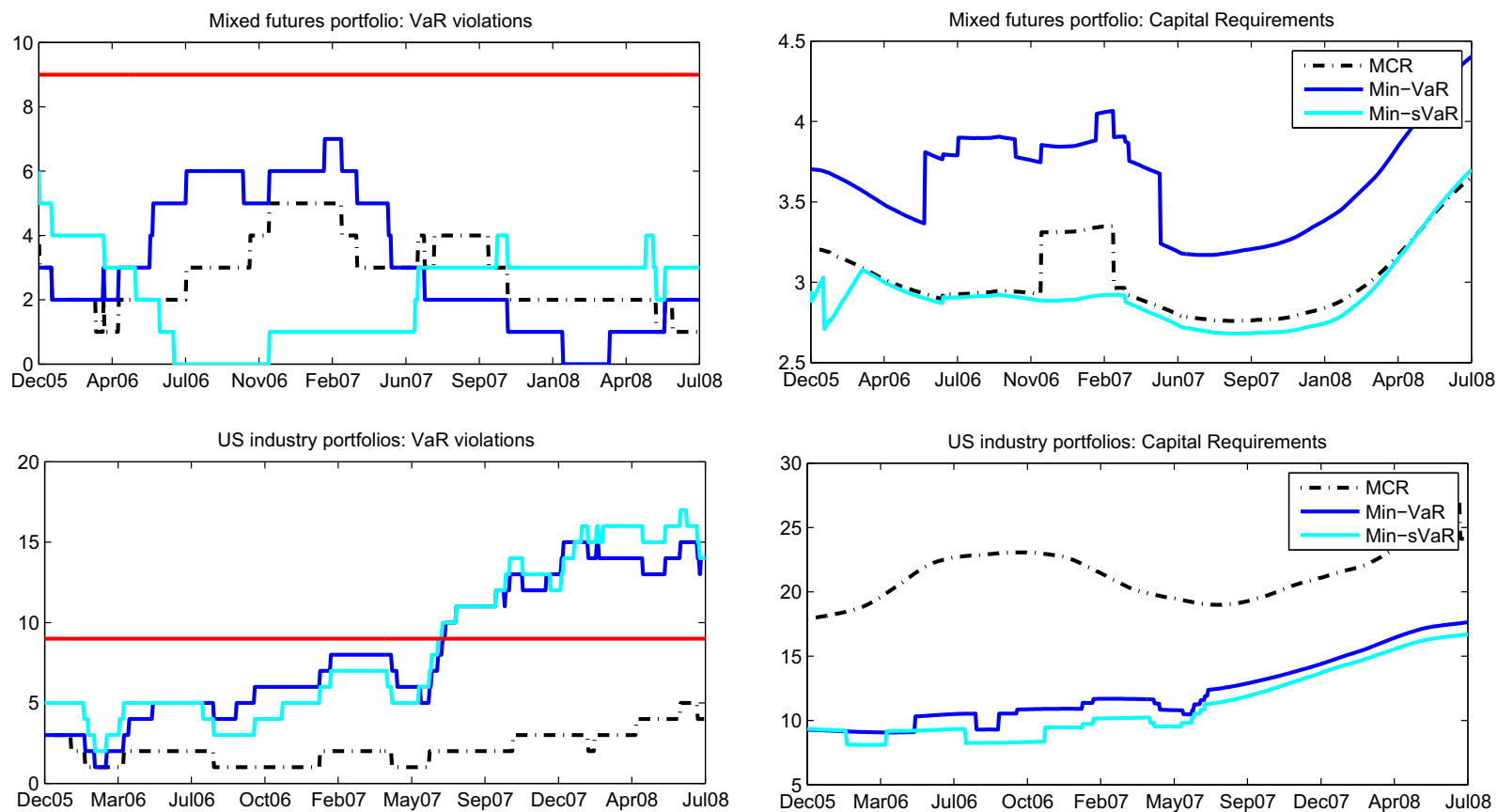

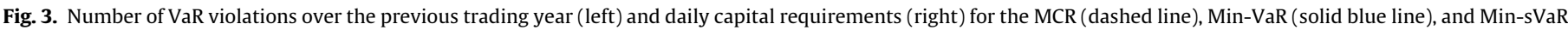

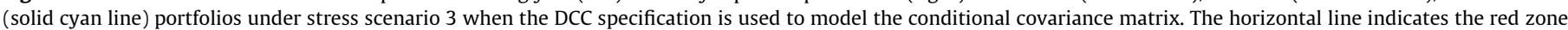

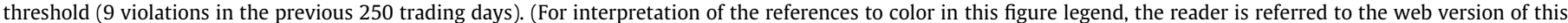
article.)

the equally weighted portfolio delivers much higher average and maximum number of $\mathrm{VaR}$ violations in comparison to the MCR and Min VaR portfolios, leading to a higher fraction of days within the red zone.

Summarizing the results in Tables 35 , the optimal MCR portfo lios outperform the benchmark portfolios in several aspects. First, the MCR portfolios achieve a better balance between capital requirement levels and the number of VaR violations in compari son to the benchmark portfolios. The average number of VaR viola tions under the MCR portfolio strategy is the lowest in the vast majority of the specifications for the conditional covariance matrix and in all stress scenarios considered. Second, and in contrast to the competing portfolio strategies, the maximum number of VaR violations for the MCR portfolio almost never exceeds the yellow zone upper bound. Third, MCR portfolios achieve a better perfor mance in terms of gross returns and Sharpe ratios in comparison to the Min VaR and Min sVaR benchmarks (at least for the US industry portfolios). Finally, turnover for the MCR portfolio is lower than for the Min VaR and Min sVaR portfolios, which together with the higher gross returns results in substantially higher break even transaction costs.

\subsection{Robustness checks}

A potential concern about the results presented above is that they could be driven by a specific choice of the portfolio target re turn, of the re balancing frequency, or of the econometric specifi cations for the expected returns. In order to rule out this possibility, in this section we briefly summarize the results from an extensive sensitivity analysis to check the robustness of the MCR portfolio's performance to changes in each of those settings. We report the detailed results of the robustness checks in a web appendix available at https://sites.google.com/site/andreportela/ MDCR_portfolios_JBF_web_appendix.pdf.

The results of the robustness checks are reassuring. First, we find that results are not sensitive to the level of the daily target return $\Xi$ in (10), which we vary between 2,4 and 6 bp (equivalent to annual target returns of 5\%,10\% and 15\%). For the futures port folio, the performance of the MCR strategy in terms of average DCR and the number of VaR violations is similar to those obtained with the benchmark policies regardless of the values of $\Xi$ considered. However, the MCR strategy delivers lower portfolio turnover, such that the breakeven transaction costs are higher in comparison to the benchmark strategies. For the US industry portfolios, the MCR strategy delivers a lower average number of VaR violations, higher average gross returns, higher Sharpe ratio, lower portfolio turnover, and higher breakeven transaction costs in comparison to the Min VaR and Min sVaR policies, irrespective of the target re turn. Finally, we conclude that an increase in the target return leads only to a less than proportional (i.e. marginal) increase in the average gross returns and in the average Sharpe ratios.

Second, the (relative) performance of the MCR strategy is not dri ven by the portfolio re balancing frequency. The results discussed above are based on the assumption that investors adjust their port folio on a daily basis. The transaction costs incurred with such frequent trading can possibly deteriorate the net portfolio perfor mance. Obviously this effect can be reduced by adjusting the port folio less frequently, such as on a weekly or monthly basis, which in fact is done in practice by many institutional investors. A draw back of re balancing the portfolio less frequently is that the portfo lio weights become outdated, which may harm its performance. We examine the performance of the MCR, Min VaR and Min sVaR port folio strategies under daily, weekly and monthly re balancing fre quencies. As expected, we find that lowering the re balancing frequency results in a substantial reduction in portfolio turnover. Other characteristics are hardly affected, neither in terms of abso lute levels nor in terms of relative values for different portfolio strategies. This holds both for the capital requirement levels and VaR violations, as well as traditional performance measures such as the Sharpe ratio.

Third, our results are robust to the model specification used for the expected returns. The VAR(1) model in (15) contains $N+N^{2}$ 
unknown coefficients. Its use for generating expected returns thus entails a large amount of estimation uncertainty for the values of $N$ considered in this paper. As a more parsimonious alternative we consider using univariate AR(1) models for each individual asset, i.e. the matrix $\Phi$ in (15) is restricted to be diagonal. A drawback of this simplification is that it ignores possibly important dynamic cross correlations among the assets in the portfolio. The results of this robustness check indicate that regardless of the specification used for the conditional covariance matrix, the MCR portfolios con tinue to perform better than the benchmark portfolios when ex pected returns are obtained from univariate AR(1) models. Compared to the results based on the unrestricted VAR(1) model, we observe that for the mixed futures portfolio, the average DCR and the average number of VaR violations tends to be higher when univariate AR(1) models are used. However, the results for the US industry portfolios are mixed. Finally, we find that in the vast majority of the cases, modeling expected returns with AR(1) mod els results in higher Sharpe ratios and higher breakeven transac tion costs compared to those originally obtained with a $\operatorname{VAR}(1)$ model.

\section{Concluding remarks}

Banks and other large financial institutions tend to overesti mate the VaR of their asset portfolios during tranquil times and to underestimate their risk in times of stressed market conditions, as documented in previous empirical studies. The VaR overestima tion during quiet periods results in prohibitive amounts of regula tory capital requirements, thus generating opportunity costs and giving rise to reputational concerns. On the other hand, the under estimation of risk levels is unattractive as it may lead to an exces sive number of VaR violations and higher than expected losses. Furthermore, the regulations in the Basel II accord impose a pen alty on the regulatory capital in case VaR exceedances occur too frequently, such that lower VaR estimates may actually increase capital requirements. The procyclicality of VaR estimates does not only have drawbacks for the banks involved, but is also unde sirable from a regulator's point of view. Given that underestima tion of the VaR during periods of turmoil appears to be widespread across banks and other financial institutions, this prac tice results in a high level of systemic risk. For this reason, recent amendments to the Basel II accord prescribe that regulatory capital should account for the downside risk under stressed market cir cumstances, in addition to the regular VaR.

In this paper we proposed a novel approach based on active portfolio selection that aims to alleviate these problems. The meth odology involves setting portfolio weights in order to minimize the level of capital requirements, but subject to a restriction on the number of historical VaR exceedances during the preceding year. Other constraints, involving, for instance, the target performance of the portfolio may be incorporated in the portfolio construction as well. An empirical application to two portfolios composed of dif ferent types of assets demonstrates that the proposed approach is able to provide a much better balance between capital requirement levels and the number of VaR violations compared to the mini mum VaR portfolio, minimum stressed $\mathrm{VaR}$, and the $1 / \mathrm{N}$ portfolio under alternative, realistic stress scenarios. This result is robust to the specifications of the expected returns and of the conditional covariance matrix, as well as to the level of target returns, and to the portfolio re balancing frequency.

\section{Acknowledgements}

We thank an anonymous referee for detailed comments and suggestions, which have led to substantial improvements in the paper. We are also grateful to Kees Bouwman, Newton da Costa Jr., Victor DeMiguel, Andreas Heinen, Erik Kole, Marcio Laurini, Mi chael McAleer, Roberto Meurer, Alfonso Novales, Pilar Poncela, Antonio Sanvicente, Timo Teräsvirta, Helena Veiga, Pedro Valls, Bernardo da Veiga, and seminar participants at Universidad Carlos III de Madrid, Econometric Institute Erasmus University Rotter dam, Insper, EESP Fundação Getúlio Vargas, and the XI Meeting of the Brazilian Finance Society for helpful comments and discus sions. André A. P. Santos acknowledges financial support from research Projects CNPq 481719/2011 3 and UFSC Funpesquisa 2010/2011 from the Brazilian Government. Francisco J. Nogales is supported by the Spanish Government through Project MTM 2010 16519. Esther Ruiz is supported by the Spanish Government ECO2009 08100.

\section{Appendix A. Supplementary data}

Supplementary data associated with this article can be found, in the online version, at http://dx.doi.org/10.1016/j.jbankfin.2012. 03.001 .

\section{References}

Aguilar, O., West, M., 2000. Bayesian dynamic factor models and portfolio allocation. Journal of Business and Economic Statistics 18, 338-357.

Alexander, C., 2009. Market Risk Analysis: Volume IV: Value at Risk Models. Wiley. Alexander, G., Baptista, A., 2002. Economic implications of using a mean-VaR model for portfolio selection: a comparison with mean-variance analysis. Journal of Economic Dynamics and Control 26, 1159-1193.

Alexander, G., Baptista, A., Yan, S., 2007. Mean-variance portfolio selection with value-at-risk constraints and discrete distributions. Journal of Banking and Finance $31,3761-3781$.

Bank for International Settlements, 2006. International Convergence of Capital Measurement and Capital Standards. Basel Committee Publications, Bank For International Settlements.

Bank for International Settlements, 2009. Revisions to the Basel II Market Risk Framework. Basel Committee Publications, Bank For International Settlements.

Barndorff-Nielsen, O., Hansen, P., Lunde, A., Shephard, N., 2008. Multivariate Realised Kernels: Consistent Positive Semi-definite Estimators of the Covariation of Equity Prices with Noise and Non-synchronous Trading. Working Paper.

Bauwens, L., Laurent, S., Rombouts, J., 2006. Multivariate GARCH models: a survey. Journal of Applied Econometrics 21, 79-109.

Berkowitz, J., O'Brien, J., 2002. How accurate are value-at-risk models at commercial banks? Journal of Finance 57, 1093-1111.

Boyd, S., Vandenbergue, L., 2004. Convex Optimization. Cambridge University Press, Cambridge.

Carriero, A., Kapetanios, G., Marcellino, M., 2009. Forecasting exchange rates with a large Bayesian VAR. International Journal of Forecasting 25, 400-417.

Chib, S., Omori, Y., Asai, M., 2009. Multivariate stochastic volatility. In: Andersen, T., Davis, R., Kreiss, J.P., Mikosch, T. (Eds.), Handbook of Financial Time Series. Springer Verlag, Berlin, pp. 365-402.

Christoffersen, P., 2009. Value-at-risk models. In: Andersen, T., Davis, R., Kreiss, J.P., Mikosch, T. (Eds.), Handbook of Financial Time Series. Springer Verlag, Berlin, pp. 753-766.

Committee of European Banking Supervisors, 2010. Aggregate Outcome of the 2010 EU Wide Stress Test Exercise Coordinated by CEBS in Cooperation with the ECB.

Cornuejols, G., Tütüncü, R., 2007. Optimization Methods in Finance. Cambridge University Press, Cambridge.

Cuoco, D., Liu, H., 2006. An analysis of VaR-based capital requirements. Journal of Financial Intermediation 15, 362-394.

Danielsson, J., Zigrand, J., 2006. On time-scaling of risk and the square-root-of-time rule. Journal of Banking and Finance 30, 2701-2713.

DeMiguel, V., Garlappi, L., Nogales, F., Uppal, R., 2009a. A generalized approach to portfolio optimization: improving performance by constraining portfolio norms. Management Science 55, 798-812.

DeMiguel, V., Garlappi, L., Uppal, R., 2009b. Optimal versus naive diversification: how inefficient is the $1 / \mathrm{N}$ portfolio strategy? Review of Financial Studies 22, 1915-1953.

DeMiguel, V., Nogales, F.J., Uppal, R., 2010. Stock Return Serial Dependence and outof-sample portfolio performance. Working Paper, London Business School.

De Pooter, M., Martens, M., van Dijk, D., 2008. Predicting the daily covariance matrix for S\&P 100 stocks using intraday data-but which frequency to use? Econometric Reviews 27, 199-229.

Diebold, F., Hickman, A., Inoue, A., Schuermann, T., 1998. Converting 1-day volatility to h-day volatility: scaling by is worse than you think. Risk 11, 104-107.

Efron, B., Gong, G., 1983. A leisurely look at the bootstrap, the jackknife, and crossvalidation. The American Statistician 37, 36-48. 
Engle, R., 2002. Dynamic conditional correlation: a simple class of multivariate generalized autoregressive conditional heteroskedasticity models. Journal of Business \& Economic Statistics 20, 339-350

Engle, R., Sheppard, K., 2001. Theoretical and Empirical Properties of Dynamic Conditional Correlation Multivariate GARCH. NBER Working Paper No. 8554.

Engle, R., Shephard, N., Sheppard, K., 2008. Fitting Vast Dimensional Time-Varying Covariance Models. Discussion Paper Series No. 403, Department of Economics, University of Oxford.

Grant, M., Boyd, S., 2008. Cvx: Matlab Software for Disciplined Convex Programming (Web Page and Software). <http://stanford.edu/boyd/cvx>.

Han, Y., 2006. Asset allocation with a high dimensional latent factor stochastic volatility model. Review of Financial Studies 19, 237-271.

Harvey, A., Ruiz, E., Shephard, N., 1994. Multivariate stochastic variance models. Review of Economic Studies 61, 247-264.

Hendricks, D., Hirtle, B., 1997. Bank capital requirements for market risk: the internal models approach. Economic Policy Review 3, 1-12.

Jagannathan, R., Ma, T., 2003. Risk reduction in large portfolios: why imposing the wrong constraints helps. Journal of Finance 58, 1651-1684.

Jorion, P., 2002. How informative are value-at-risk disclosures? Accounting Review, 911-931.

Ledoit, O., Wolf, M., 2003. Improved estimation of the covariance matrix of stock returns with an application to portfolio selection. Journal of Empirical Finance $10,603-621$.

Ledoit, O., Wolf, M., 2004. Honey, I shrunk the sample covariance matrix. Journal of Portfolio Management 30, 110-119.

Ledoit, O., Wolf, M., 2008. Robust performance hypothesis testing with the sharpe ratio. Journal of Empirical Finance 15, 850-859.
McAleer, M., Amaral, T.P., Jiménez-Martin, J.A., 2010. A decision rule to minimize daily capital charges in forecasting value-at-risk. Journal of Forecasting 29, 617 634.

Nocedal, J., Wright, S., 1999. Numerical Optimization. Springer Verlag, Berlin.

Pérignon, C., Deng, Z., Wang, Z., 2008. Do banks overstate their value-at-risk? Journal of Banking \& Finance 32, 783-794.

Pesaran, M., Schuermann, T., Smith, L., 2009. Forecasting economic and financia variables with global VARs. International Journal of Forecasting 25, 642-675.

Politis, D., Romano, J., 1994. The stationary bootstrap. Journal of the American Statistical Association 89, 1303-1313.

PTrignon, C., Smith, D., 2010. Diversification and value-at-risk. Journal of Banking \& Finance 34, 55-66.

Qi, H., Sun, D., 2010. Correlation stress testing for value-at-risk: an unconstrained convex optimization approach. Computational Optimization and Applications 45, 427-462.

Santos, A.A.P., Nogales, F., Ruiz, E., 2009. Comparing Univariate and Multivariate Models to Forecast Portfolio Value-at-Risk. Working Paper 09-72, Department of Statistics, Universidad Carlos III de Madrid.

Sentana, E. 2003. Mean-variance portfolio allocation with a value at risk constraint. Revista de Economfa Financiera 1, 4-14.

Sharpe, W., 1963. A simplified model for portfolio analysis. Management Science 9 277-293.

Sheppard, K., 2003. Multi-step estimation of multivariate GARCH models. In: Proceedings of the International ICSC. Symposium: Advanced Computing in Financial Markets.

Silvennoinen, A., TerSsvirta, T, 2009. Multivariate GARCH models. In: Andersen, T. Davis, R., Kreiss, J.P., Mikosch, T. (Eds.), Handbook of Financial Time Series. Springer Verlag, Berlin, pp. 201-229. 